\title{
A parallel discontinuous Galerkin/cohesive-zone computational framework for the simulation of fracture in shear-flexible shells
}

\author{
Brandon L. Talamini, Raúl Radovitzky* \\ Department of Aeronautics and Astronautics \\ Massachusetts Institute of Technology \\ Cambridge, MA 02139
}

\begin{abstract}
We propose a computational framework for the simulation of deformation and fracture in shells that is well suited to situations with widespread damage and fragmentation due to impulsive loading. The shell is modeled with a shear-flexible theory and discretized with a discontinuous Galerkin finite element method, while fracture is represented with a cohesive zone model on element edges. A key feature of the method is that the underlying shear-flexible shell theory enables the description of transverse shear fracture modes, in addition to the in-plane and bending modes accessible to Kirchhoff-Love thin shell formulations. This is especially important for impulsive loading conditions, where shear-off failure near stiffeners and supports is common. The discontinuous Galerkin formulation inherits the scalability properties demonstrated previously for large-scale simulation of fracture in solids, while avoiding artificial elastic compliance issues that are common in other cohesive model approaches. We demonstrate the ability of the framework to capture the transverse shear fracture mode through numerical examples, and the parallel computation capabilities of the method through the simulation of explosive decompression of the skin of a full-scale passenger aircraft fuselage.
\end{abstract}

Keywords: shells, fracture, discontinuous Galerkin, cohesive zone, parallel computing

\section{Introduction}

Fracture is a critical concern in the engineering of many shell structures, e.g., blast protection of ships and submarines, ship grounding, vehicle crashworthiness, and accident reconstruction and analysis for passenger aircraft. Advances in computing power notwithstanding, direct simulation of shell structures with three dimensional continuum methods is impractical, particularly in cases when the structure is large, fracture is widespread, and the failure locations are not known a priori. Shell theory remains the method of choice to attack these problems. In this work, we develop a computational framework for the simulation of deformation and fracture in shells that is well suited to simulating scenarios with massive damage and fragmentation under highly impulsive loading. The consideration of fracture driving forces is quite general and comprises the full range of modes possible for shells: in plane forces, bending, and transverse shear.

We model fracture as a process of surface decohesion in the style of Barenblatt $[1,2]$. In particular, our approach belongs to the family of methods that implement the cohesive fracture concept through interface elements that allow fracture on element boundaries. This technique was originally developed for fracture in solids (see, e.g., [3, 4] and the review of the technique in [5]), and was adapted to the special case of shells in the last decade $[6,7]$. Our approach builds on this work in a number of ways. The framework here is based on a discontinuous Galerkin (DG) spatial discretization using a finite deformation, Reissner-Mindlin

\footnotetext{
* Corresponding author.

Email addresses: talamini@mit.edu (Brandon L. Talamini), rapa@mit.edu (Raúl Radovitzky)
} 
element that we recently proposed [8]. With this discretization scheme, we are able to take advantage of the paradigm of $[5,9,10]$ combining a DG method with interface element-based cohesive fracture to achieve a method that is both scalable in parallel computation and also free of artificial elastic compliance in the cohesive zone constitutive behavior. For scalability, the key property is that the shell elements in the DG method do not share degrees of freedom. Thus activating cohesive zones on element interfaces does not require the creation of new degrees of freedom, nor the modification of the finite element data structures, even when cracks grow across or along CPU sub-domain boundaries. The DG method naturally contains boundary integrals on element edges that may conveniently be replaced with the cohesive behavior once the critical stress for crack nucleation has been reached. Regarding the artificial elastic compliance, the DG scheme rigorously enforces compatibility between elements in a weak manner prior to activation of cohesive fracture, sidestepping the need to enforce compatibility with an artificial elastic regime in the cohesive law. One benefit of this is that the consistency error of the penalty method is avoided (see [11]) and optimal convergence rates are preserved; furthermore, larger time steps may be taken during explicit time integration as the penalty stiffness tends to dominate the stable time step limit in the artificial compliance method [12].

The aforementioned benefits of our framework are shared by the DG shell method of Becker et al. [13, 14]. The present work is distinguished by its shear-flexible shell formulation, in contrast to the Kirchhoff-Love formulation used in $[6,13,14]$. The additional shearing deformation mode of our proposed approach enables the simulation of through-thickness shear fracture (in addition, of course, to the in-plane and bending modes). An obvious application for this capability is the simulation of relatively thick shells; another, perhaps less obvious, is in modeling of highly impulsive loads for both thick and thin shells. High-rate loadings, such as impacts and blasts, increase the influence of transverse shearing on the response, even in shells that might be considered "thin" in a quasi-static scenario. When the loading is severe and the duration is short compared to the flexural response time of the structure, the effective span bearing the load is shortened, and shear-off fracture at stiffeners and supports may occur before the shell bends. This phenomenon is illustrated vividly by the beam blast experiments of Menkes and Opat [15] (see Figure 1). In Section 6 we simulate these experiments to demonstrate that our framework can capture this effect.

As an added benefit, the proposed formulation inherits the locking-free behavior of the underlying DG method (see [8]). Thus locking is avoided without requiring additional techniques, such as reduced integration rules or mixed methods.

To complete the framework, we construct a simple cohesive zone model specially designed for shell fracture. Our method is based on the developments in [7], in which cohesive laws were formulated directly in terms of stress resultants and the conjugate generalized displacements. This approach takes full advantage of the dimensional reduction made in the underlying shell theory - the alternative approach is to apply expensive numerical integration of three-dimensional constitutive laws through the shell thickness. The cohesive zone constitutive relations in [7] jointly consider in-plane forces, transverse forces, and bending. We extend this work by removing the artificial elastic compliance and including an additional twisting mode for full generality with the Reissner-Mindlin shell kinematics.

In [7] it was shown that for many ductile fracture problems in shells it is possible to satisfactorily lump the cohesive zone constitutive laws into resultant form, even in the presence of 3D effects such as slant fracture and crack tunneling, provided that the cohesive laws are calibrated to the shell thickness in addition to the behavior of the material. Our proposed method should enjoy the same property. The framework herein embedding the cohesive zone model is also general and may be used with interface constitutive laws specially adapted to different problem types. For example, important improvements in plate and shell cohesive zone constitutive models for ductile fracture have recently been proposed (cf. [16-20]); these models could be deployed in the framework proposed here.

The structure of the paper is as follows: we review the governing equations of the shell theory in Section 2. In Section 3 we lay out a theory of resultant-based cohesive fracture in shells including the effects of transverse shear, and then propose a particular form of the cohesive zone constitutive relations (i.e., the traction-separation law). Section 4 is devoted to the derivation of the discrete equations of motion and a simple extension of the Newmark algorithm for their integration in time. In section 5, we discuss special considerations for the implementation of the fracture model within the DG discretization in order to promote its adoption and further development. Numerical examples are presented in Section 6, including simulation 


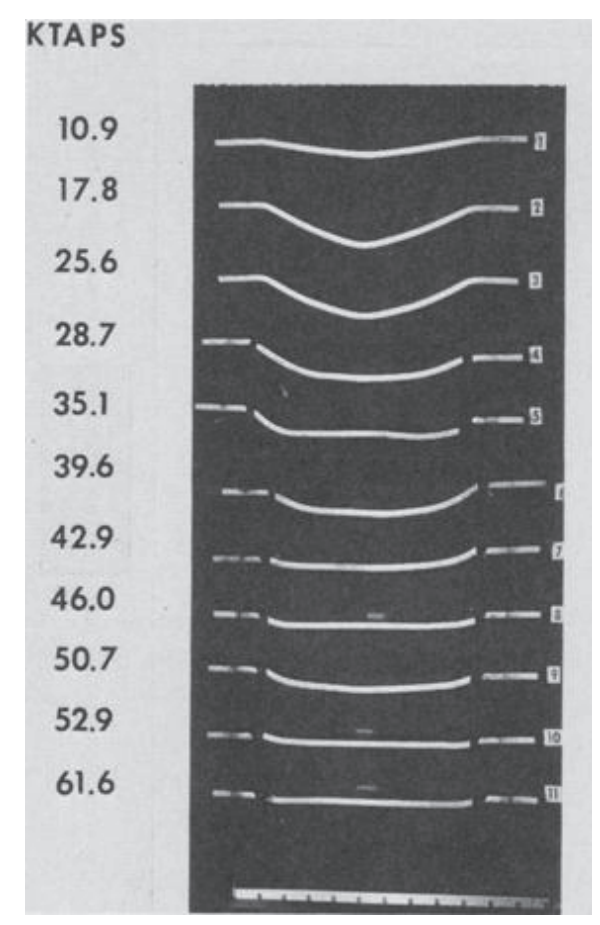

Figure 1: Impulsively loaded aluminum beam experiments of Menkes and Opat (1973). The beams were clamped at both ends, and plastic explosives of varying thickness were attached to the beam surface and detonated to control the impulse delivered. These photograph shows the transition from tensile failure at low impulses (top) to shear-off at high impulses (bottom). Image source: Menkes and Opat [15]

of the above mentioned beam blast experiments [15] that illustrate the increased importance of transverse shearing on fracture in highly impulsive loadings. Finally we conclude with some remarks in Section 7.

\section{Governing equations}

The adopted shell formulation is that of a classical single director Cosserat surface, in the form developed by Simo and Fox [21]. For completeness, and to fix notation, the theory is summarized in this section.

\subsection{Kinematics}

The parameterization of the shell mid-surface $\mathcal{S}$ is given by a smooth injective map $\varphi: \mathcal{A} \subset \mathbb{R}^{2} \rightarrow \mathbb{R}^{3}$. The boundary of the mid-surface is indicated by $\partial \mathcal{S}$. Material points in the shell body are located relative to the mid-surface with the director vector field $\tilde{\mathbf{t}}: \mathcal{S} \rightarrow S^{2}$, where $S^{2}$ is the unit sphere. The basic kinematic assumption of the theory is that points in the shell body $\mathcal{B}_{t}=\mathcal{S} \times(-h / 2, h / 2)$ at time $t$ are given by the map

$$
\mathbf{x}=\mathbf{\Phi}_{t}\left(\xi^{1}, \xi^{2}, \xi^{3}\right)=\boldsymbol{\varphi}\left(\xi^{1}, \xi^{2}, t\right)+\xi^{3} \mathbf{t}\left(\xi^{1}, \xi^{2}, t\right), \quad \xi^{3} \in(-h / 2, h / 2), t \in[0, T]
$$

where $h$ is the thickness of the shell, and $\mathbf{t}:=\tilde{\mathbf{t}} \circ \varphi$ is the parameterization of the director. The reference configuration is given similarly by

$$
\mathbf{x}^{0}=\boldsymbol{\Phi}^{0}\left(\xi^{1}, \xi^{2}, \xi^{3}\right)=\varphi^{0}\left(\xi^{1}, \xi^{2}\right)+\xi^{3} \mathbf{t}^{0}\left(\xi^{1}, \xi^{2}\right), \quad \xi^{3} \in(-h / 2, h / 2)
$$

\footnotetext{
${ }^{1}$ In general, a collection of parameterizations are needed to cover $\mathcal{S}$, but for notational simplicity they are not individually indexed.
} 
Here and in the following, kinematic quantities in the reference configuration are distinguished from their counterparts in the current configuration by labeling them with a superscript 0 . The reference configuration is taken to be that occupied by the shell at the initial time,

$$
\begin{aligned}
\varphi^{0} & =\left.\varphi\right|_{t=0} \\
\mathbf{t}^{0} & =\left.\mathbf{t}\right|_{t=0}
\end{aligned}
$$

It follows from the choice of the unit sphere as the director manifold and the assumed invariance of $h$ that the shell is inextensible in the thickness direction. The motion of the shell is given by the composition $\chi\left(\xi^{1}, \xi^{2}, \xi^{3}, t\right):=\boldsymbol{\Phi}_{t} \circ\left[\mathbf{\Phi}^{0}\right]^{-1}$. The kinematics are illustrated in Figure 2.

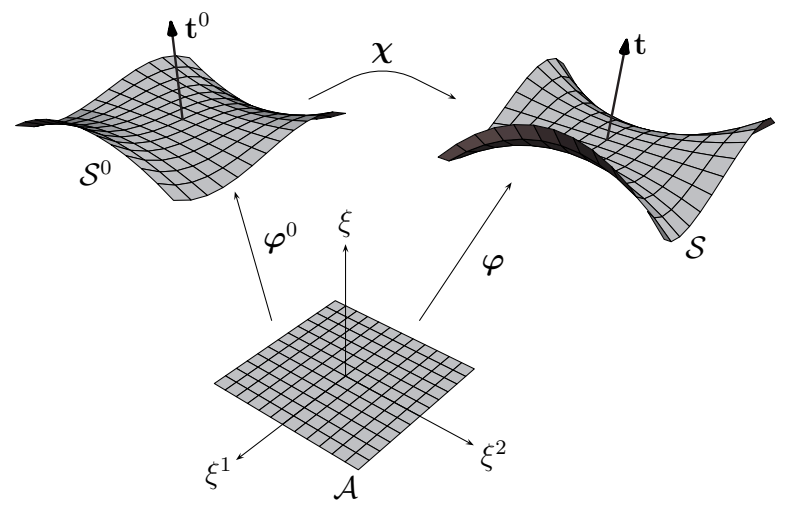

Figure 2: Reference and current configurations of the shell.

The covariant basis vectors in $\mathcal{S}^{0}$ and $\mathcal{S}$ are

$$
\mathbf{a}_{\alpha}^{0}:=\partial_{\alpha} \boldsymbol{\varphi}^{0} \quad \mathbf{a}_{\alpha}:=\partial_{\alpha} \boldsymbol{\varphi},
$$

where the notation $\partial_{\alpha}:=\partial / \partial \xi^{\alpha}$ is used to indicate partial differentiation with respect to the parametric coordinates.

The mid-surface measures in the reference and current configurations are $d \mathcal{S}^{0}=\bar{j}^{0} d \mathcal{A}$ and $d \mathcal{S}=\bar{j} d \mathcal{A}$, with $d \mathcal{A}:=d \xi^{1} d \xi^{2}$ and

$$
\bar{j}^{0}=\mathbf{t}^{0} \cdot\left(\mathbf{a}_{1}^{0} \times \mathbf{a}_{2}^{0}\right) \quad \bar{j}=\mathbf{t} \cdot\left(\mathbf{a}_{1} \times \mathbf{a}_{2}\right)
$$

The Jacobian of the mid-surface deformation is $\bar{J}=\bar{j} / \bar{j}^{0}>0$.

Differentiation of quantities with respect to time (holding the material point fixed) is indicated by Newton's over-dot notation. The director velocity field $\dot{\mathbf{t}}$ belongs to the tangent space of the unit sphere $T_{\mathbf{t}} S^{2}$, which has the explicit representation

$$
T_{\mathbf{t}} S^{2}=\left\{\mathbf{w} \in \mathbb{R}^{3}: \mathbf{w} \cdot \mathbf{t}=0\right\}
$$

In Section 4.1, we describe an interpolation such that this geometric structure is maintained in the discrete setting.

The shell is subject to the essential boundary conditions

$$
\begin{aligned}
\boldsymbol{\varphi}\left(\xi^{\alpha}, t\right) & =\overline{\boldsymbol{\varphi}}\left(\xi^{\alpha}, t\right), \quad \text { on } \partial_{\varphi} \mathcal{A} \times[0, T] \\
\mathbf{t}\left(\xi^{\alpha}, t\right) & =\overline{\mathbf{t}}\left(\xi^{\alpha}, t\right), \quad \text { on } \partial_{t} \mathcal{A} \times[0, T]
\end{aligned}
$$

where $\partial_{\varphi} \mathcal{A}$ and $\partial_{t} \mathcal{A}$ are open subsets of $\partial \mathcal{A}$. The configuration space of the shell at any time $t \in[0, T]$ is thus

$$
\mathbb{X}_{t}=\left\{(\varphi(\cdot, t), \mathbf{t}(\cdot, t)) \in \mathcal{A} \rightarrow \mathbb{R}^{3} \times S^{2}:\left.\varphi(\cdot, t)\right|_{\partial_{\varphi} \mathcal{A}}=\bar{\varphi},\left.\mathbf{t}(\cdot, t)\right|_{\partial_{t} \mathcal{A}}=\overline{\mathbf{t}}\right\}
$$




\subsection{Weak form of the equations of motion}

At any fixed time, the shell motion is imagined to be perturbed by virtual velocities, chosen from the space

$$
\mathbb{V}=\left\{(\boldsymbol{\eta}, \mathbf{w}): \mathcal{A} \rightarrow \mathbb{R}^{3} \times T_{\mathbf{t}} S^{2}:\left.\boldsymbol{\eta}\right|_{\partial_{\varphi} \mathcal{A}}=\mathbf{0},\left.\mathbf{w}\right|_{\partial_{t} \mathcal{A}}=\mathbf{0}\right\}
$$

The principle of virtual power states

$$
\delta \mathcal{P}_{\text {ext }}(\boldsymbol{\eta}, \mathbf{w})-\delta \mathcal{P}_{\text {int }}(\boldsymbol{\varphi}, \mathbf{t} ; \boldsymbol{\eta}, \mathbf{w})=\delta \dot{\mathcal{K}}(\boldsymbol{\varphi}, \mathbf{t} ; \boldsymbol{\eta}, \mathbf{w}), \quad \forall(\boldsymbol{\eta}, \mathbf{w}) \in \mathbb{V}
$$

where $\delta \mathcal{P}_{\text {ext }}$ is the virtual power of the external loading, $\delta \mathcal{P}_{\text {int }}$ is the internal virtual power, and $\delta \dot{\mathcal{K}}$ is the virtual kinetic energy storage rate.

The external virtual power has the form

$$
\delta \mathcal{P}_{\text {ext }}(\boldsymbol{\eta}, \mathbf{w})=\int_{\mathcal{S}^{0}}(\mathbf{f} \cdot \boldsymbol{\eta}+\boldsymbol{\tau} \cdot \mathbf{w}) d \mathcal{S}^{0}+\int_{\partial_{N} \mathcal{A}} \overline{\mathbf{n}} \cdot \boldsymbol{\eta} \bar{j}^{0} d s+\int_{\partial_{M} \mathcal{A}} \overline{\mathbf{m}} \cdot \mathbf{w} \bar{j}^{0} d s .
$$

The body force terms $\mathbf{f}$ and $\boldsymbol{\tau}$ are the effective distributed forces and torques on the mid-surface, respectively. The symbols $\overline{\mathbf{n}}$ and $\overline{\mathbf{m}}$ represent prescribed resultant tractions. By $d s$ we denote the differential element of arc length in $\mathcal{A}$. We assume that the boundary of the shell (if any) is partitioned into regions of essential and natural boundary conditions according to

$$
\begin{aligned}
\partial_{\varphi} \mathcal{A} \cap \partial_{N} \mathcal{A}=\varnothing, & \partial_{\varphi} A \cup \partial_{N} \mathcal{A}=\partial \mathcal{A}, \\
\partial_{\mathbf{t}} \mathcal{A} \cap \partial_{M} \mathcal{A}=\varnothing, & \partial_{\mathbf{t}} \mathcal{A} \cup \partial_{M} \mathcal{A}=\partial \mathcal{A} .
\end{aligned}
$$

The internal virtual power is decomposed into two parts,

$$
\delta \mathcal{P}_{\text {int }}=\delta \mathcal{P}_{\mathrm{s}}+\delta \mathcal{P}_{\mathrm{c}}
$$

the stress power $\delta \mathcal{P}_{\mathrm{s}}$ and an additional cohesive zone power $\delta \mathcal{P}_{\mathrm{c}}$ associated with fracture (the cohesive zone kinematics and power expenditure are discussed in the next section). Following [21], the virtual stress power is taken to be

$$
\delta \mathcal{P}_{\mathrm{s}}(\boldsymbol{\varphi}, \mathbf{t} ; \boldsymbol{\eta}, \mathbf{w})=\int_{\mathcal{S}^{0}}\left(\mathbf{n}^{\alpha} \cdot \partial_{\alpha} \boldsymbol{\eta}+\mathbf{m}^{\alpha} \cdot \partial_{\alpha} \mathbf{w}+\mathbf{l} \cdot \mathbf{w}\right) d \mathcal{S}^{0},
$$

where $\mathbf{n}^{\alpha}, \mathbf{m}^{\alpha}$, and $\mathbf{l}$ are resultants that can be related the standard three-dimensional stress tensors of continuum mechanics through appropriate integrals over the shell thickness. ${ }^{2}$ Applying the typical terminology, $\mathbf{n}^{\alpha}$ are the stress resultants, $\mathbf{m}^{\alpha}$ are the stress couple resultants, and $\mathbf{l}$ is the through-thickness stress resultant. The dependence of the virtual internal power $\delta \mathcal{P}_{\mathrm{S}}$ on $\varphi$ and $\mathbf{t}$ occurs implicitly through the constitutive relations for the stress resultants.

The kinetic energy $\mathcal{K}$ is taken to be composed of the quadratic forms

$$
\mathcal{K}=\int_{\mathcal{S}^{0}}\left(\frac{1}{2} \bar{\rho}^{0}\|\dot{\boldsymbol{\varphi}}\|^{2}+\frac{1}{2} \bar{I}^{0}\|\dot{\mathbf{t}}\|^{2}\right) d \mathcal{S}^{0}
$$

where $\bar{\rho}^{0}=h \rho^{0}$ and $\bar{I}=\rho^{0} h^{3} / 12$, and $\rho^{0}$ is the mass density in the reference configuration. The virtual kinetic energy storage rate is therefore

$$
\delta \dot{\mathcal{K}}(\boldsymbol{\varphi}, \mathbf{t} ; \boldsymbol{\eta}, \mathbf{w})=\int_{\mathcal{S}_{0}}\left(\bar{\rho}^{0} \ddot{\boldsymbol{\varphi}} \cdot \boldsymbol{\eta}+\bar{I}^{0} \ddot{\mathbf{t}} \cdot \mathbf{w}\right) d \mathcal{S}^{0} .
$$

\footnotetext{
${ }^{2}$ The stress resultants used in this work are scaled by a factor of $\bar{j} / \bar{j}^{0}$ relative to those used by Simo and Fox [21]. That is, the resultants here are Kirchhoff resultants instead of Cauchy resultants.
} 


\section{Resultant-based cohesive zone model of fracture}

From the outset, we restrict attention to through-thickness cracks. As stated in the introduction, one of the goals of this work is to capture the transverse shear failure modes, shown schematically in Figure 3c and $3 \mathrm{~d}$, in addition to the more commonly modeled in-plane modes (3a and $3 \mathrm{~b})$. We cast the cohesive zone framework directly in resultant form, as opposed to numerical integration of three-dimensional quantities through the thickness (cf. [6]), to take full advantage of the dimensional reduction of shell theory and increase computational efficiency.

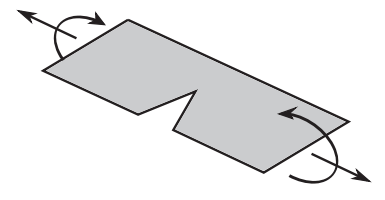

(a)

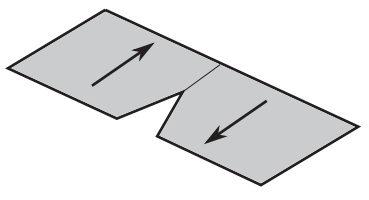

(b)

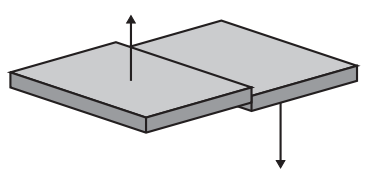

(c)

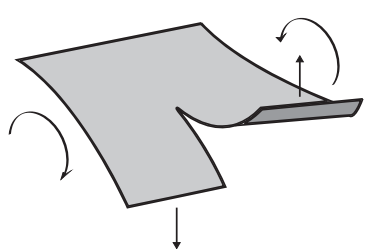

(d)

Figure 3: Basic fracture modes of thin bodies. The modes in (a) and (b) may be modeled with Kirchhoff-Love shells. The transverse shear driven modes in (c) and (d) require a shear-flexible shell theory.

A cohesive boundary $\Gamma_{\mathrm{c}}$ is supposed in the shell reference mid-surface over which the kinematic fields suffer jump discontinuities; see Figure 4. Arbitrarily labeling the two sides of the cohesive boundary $\Gamma_{\mathrm{c}}^{+}$and $\Gamma_{\mathrm{c}}^{-}$, the cohesive boundary is oriented with an in-plane normal vector $\nu$ that points from $\Gamma_{\mathrm{c}}^{-}$to $\Gamma_{\mathrm{c}}^{+}$. The notation $\llbracket \bullet$ will be used to denote the jump in a field, defined as

$$
\llbracket \bullet \rrbracket=\bullet-\bullet+
$$

where the superscript identifies which side of the edge the restriction of the field is taken, e.g., $\mathbf{v}^{-}=\left.\mathbf{v}\right|_{\Gamma_{c}^{-}}$.

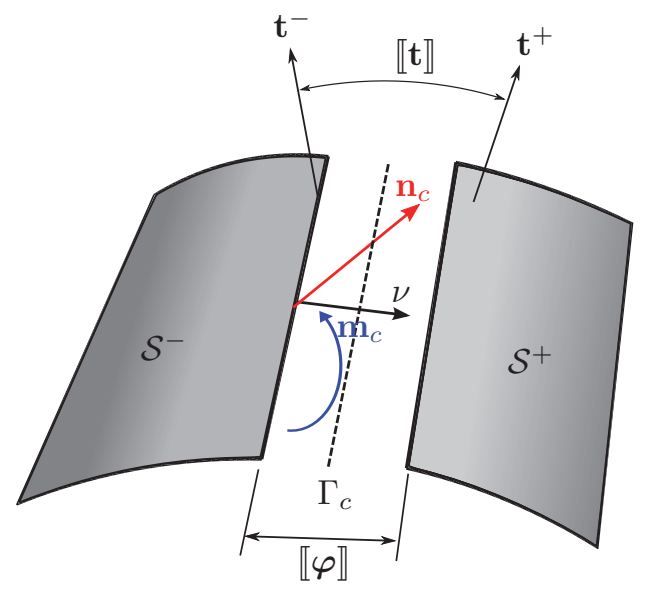

Figure 4: Cohesive surface with resultant tractions.

An additional expenditure of internal power associated to the cohesive surface is assumed in the form

$$
\mathcal{P}_{\mathrm{c}}=\int_{\Gamma_{\mathrm{c}}}\left(\mathbf{n}_{\mathrm{c}} \cdot \llbracket \dot{\boldsymbol{\varphi}} \rrbracket+\mathbf{m}_{\mathrm{c}} \cdot \llbracket \dot{\mathbf{t}} \rrbracket\right) \bar{j}^{0} d s
$$

where $\mathbf{n}_{\mathrm{c}}$ and $\mathbf{m}_{\mathrm{c}}$ are resultant tractions (force per unit undeformed length and force couple per unit undeformed length) conjugate to the virtual velocity jumps, to be specified by a cohesive law. Note that 
both $\llbracket \varphi \rrbracket$ and $\mathbf{n}_{\mathrm{c}}$ may have out-of-plane components, as necessary to describe transverse shear failure. The virtual power of the cohesive zone is therefore given by

$$
\delta \mathcal{P}_{\mathrm{c}}(\boldsymbol{\varphi}, \mathbf{t} ; \boldsymbol{\eta}, \mathbf{w})=\int_{\Gamma_{\mathrm{c}}}\left(\mathbf{n}_{\mathrm{c}} \cdot \llbracket \boldsymbol{\eta} \rrbracket+\mathbf{m}_{\mathrm{c}} \cdot \llbracket \mathbf{w} \rrbracket\right) \bar{j}^{0} d s
$$

A free energy per unit length in the reference configuration is postulated, denoted $\phi$, which is subject to the surface free energy imbalance

$$
\mathbf{n}_{c} \cdot \llbracket \dot{\boldsymbol{\varphi}} \rrbracket+\mathbf{m}_{c} \cdot \llbracket \dot{\mathbf{t}} \rrbracket-\dot{\phi} \geq 0
$$

Constitutive equations must be prescribed for the free energy and the generalized tractions. These are assumed to be in the fairly general form

$$
\begin{aligned}
\phi & =\hat{\phi}(\llbracket \boldsymbol{\varphi} \rrbracket, \llbracket \mathbf{t} \rrbracket,\{\mathbf{z}\}) \\
\mathbf{n}_{\mathrm{c}} & =\hat{\mathbf{n}}_{\mathrm{c}}(\llbracket \boldsymbol{\varphi} \rrbracket, \llbracket \mathbf{t} \rrbracket,\{\mathbf{z}\}) \\
\mathbf{m}_{\mathrm{c}} & =\hat{\mathbf{m}}_{\mathrm{c}}(\llbracket \boldsymbol{\varphi} \rrbracket, \llbracket \mathbf{t} \rrbracket,\{\mathbf{z}\})
\end{aligned}
$$

where $\{\mathbf{z}\}$ is a suitable set of internal variables, which may be of scalar or tensorial nature. Applying the standard arguments of the Coleman-Noll procedure, it is found that the constitutive functions must be related through

$$
\hat{\mathbf{n}}_{\mathrm{c}}(\llbracket \boldsymbol{\varphi} \rrbracket, \llbracket \mathbf{t} \rrbracket,\{\mathbf{z}\})=\frac{\partial \hat{\phi}(\llbracket \varphi \rrbracket, \llbracket \mathbf{t} \rrbracket,\{\mathbf{z}\})}{\partial \llbracket \boldsymbol{\varphi} \rrbracket} \quad \hat{\mathbf{m}}_{\mathrm{c}}(\llbracket \varphi \rrbracket, \llbracket \mathbf{t} \rrbracket,\{\mathbf{z}\})=\frac{\partial \hat{\phi}(\llbracket \varphi \rrbracket, \llbracket \mathbf{t} \rrbracket,\{\mathbf{z}\})}{\partial \llbracket \mathbf{t} \rrbracket},
$$

The remaining term gives the dissipation density on the cohesive edges $\mathcal{D}_{\mathrm{c}}$, which must obey the inequality

$$
\mathcal{D}_{\mathrm{c}}=-\frac{\partial \hat{\phi}(\llbracket \boldsymbol{\varphi} \rrbracket, \llbracket \mathbf{t} \rrbracket,\{\mathbf{z}\})}{\partial\{\mathbf{z}\}} \cdot\{\dot{\mathbf{z}}\} \geq 0
$$

\subsection{Specialization of the cohesive zone constitutive relations}

We adopt cohesive zone constitutive equations similar in form to the stress resultant approach proposed by Zavattieri [7]. This approach extends the scalar effective opening displacement cohesive zone model of Camacho and Ortiz [4] for fracture in solids to the realm of shells. Through this approach, the computational framework can model the effects of both transverse shear and in-plane forces on fracture in a simple way.

To begin, kinematic jumps are decomposed into normal and shearing parts

$$
\begin{aligned}
\delta_{N} & =\llbracket \boldsymbol{\varphi} \rrbracket \cdot \boldsymbol{\nu} \\
\boldsymbol{\delta}_{S} & =(\mathbf{1}-\boldsymbol{\nu} \otimes \boldsymbol{\nu}) \llbracket \boldsymbol{\varphi} \rrbracket \\
\theta_{N} & =\llbracket \mathbf{t} \rrbracket \cdot \boldsymbol{\nu} \\
\boldsymbol{\theta}_{S} & =(\mathbf{1}-\boldsymbol{\nu} \otimes \boldsymbol{\nu}) \llbracket \mathbf{t} \rrbracket
\end{aligned}
$$

Note that $\boldsymbol{\delta}_{S}$ can have a transverse opening component, which corresponds precisely to the failure mode we are striving to capture. Also note that due to the kinematics of the director field, the director jump will have no component along the director itself. In the following, we will write $\delta_{S}:=\left\|\boldsymbol{\delta}_{S}\right\|$ and $\theta_{S}:=\left\|\boldsymbol{\theta}_{S}\right\|$ for the magnitudes of the sliding components of the kinematic jumps.

Using the definitions in (19), we define a scalar effective opening displacement

$$
\delta:=\sqrt{\delta_{N}^{2}+\left(\alpha_{1} \delta_{S}\right)^{2}+\left(\alpha_{2} h \theta_{N}\right)^{2}+\left(\alpha_{3} h \theta_{S}\right)^{2}}
$$

where $\left\{\alpha_{1}, \alpha_{2}, \alpha_{3}\right\}$ are parameters introduced to weight the shear and rotational modes relative to the the in-plane normal crack opening mode. (The shell thickness $h$ is included with the angular jumps to make the parameters dimensionless). Let us remark that in this definition, our model departs slightly from [7] by 
including the $\boldsymbol{\theta}_{S}$ component of the director jump. Our motivation for including it is so that jumps in every kinematic degree of freedom are opposed by cohesive forces upon nucleation of new cohesive surface.

It is then assumed that the free energy depends on the kinematic jumps only through the scalar effective opening displacement,

$$
\hat{\phi}\left(\llbracket \varphi \rrbracket, \llbracket \mathbf{t} \rrbracket,\left\{z_{A}\right\}\right)=\bar{\phi}\left(\delta, \delta_{\max }\right),
$$

where $\delta_{\max }$, the sole internal variable, is the maximum value of the effective opening displacement attained up to the current time. The quantity

$$
n:=\frac{\partial \bar{\phi}\left(\delta, \delta_{\max }\right)}{\partial \delta}
$$

is an effective scalar traction conjugate to $\delta$. The cohesive tractions follow from (17):

$$
\begin{aligned}
\overline{\mathbf{n}}_{\mathrm{c}}\left(\delta, \delta_{\max }\right) & =\frac{n}{\delta}\left(\delta_{N} \boldsymbol{\nu}+\alpha_{1}^{2} \boldsymbol{\delta}_{S}\right) \\
\overline{\mathbf{m}}_{\mathrm{c}}\left(\delta, \delta_{\max }\right) & =\frac{n}{\delta}\left(\alpha_{2}^{2} h^{2} \theta_{N} \boldsymbol{\nu}+\alpha_{3}^{2} h^{2} \boldsymbol{\theta}_{S}\right)
\end{aligned}
$$

Note that the cohesive resultant tractions have components in the transverse direction, as desired.

The effective scalar traction law is envisioned to unload to the origin, as is common in effective opening displacement approaches (cf. [4]). Reloading occurs elastically until the maximum effective opening displacement is reached (Figure 5$)$. To this end, we introduce a strength function, $S\left(\delta_{\max }\right)$ that limits the effective scalar traction. The behavior in Figure 5 is realized by defining the free energy as

$$
\bar{\phi}\left(\delta, \delta_{\max }\right)=\frac{1}{2}\left(\frac{S\left(\delta_{\max }\right)}{\delta_{\max }}\right) \delta^{2} .
$$

The corresponding effective scalar traction is

$$
n=\frac{\partial \bar{\phi}\left(\delta, \delta_{\max }\right)}{\partial \delta}=\frac{\delta}{\delta_{\max }} S\left(\delta_{\max }\right)
$$

When the cohesive zone is actively loading, that is, when $\delta=\delta_{\max }$ and $\dot{\delta}>0$, then $n=S\left(\delta_{\max }\right)$. During unloading or reloading, where $\delta<\delta_{\max }$, the effective scalar traction decreases or increases on the line between the origin and the current strength $S\left(\delta_{\max }\right)$. (Both (24) and (25) are understood to hold in the sense of the right-handed limit as $\delta$ and $\delta_{\max }$ approach zero).

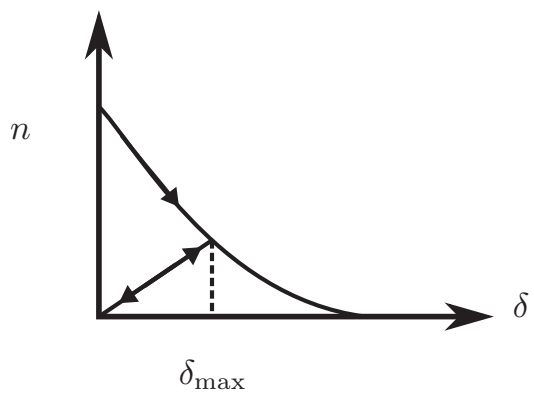

Figure 5: Schematic of effective scalar traction showing unloading to the origin.

From (18) the cohesive zone dissipation density is

$$
\mathcal{D}_{\mathrm{c}}=-\frac{\partial \bar{\phi}\left(\delta, \delta_{\max }\right)}{\partial \delta_{\max }} \dot{\delta}_{\max }=-\frac{1}{2}\left(\frac{\delta}{\delta_{\max }}\right)^{2}\left(S^{\prime} \delta_{\max }-S\right) \dot{\delta}_{\max } \quad \text { when } \dot{\delta}_{\max }>0
$$

where $S^{\prime}$ signifies $d S\left(\delta_{\max }\right) / d \delta_{\max }$. Since by definition $\dot{\delta}_{\max }=0$ if $\delta \neq \delta_{\max }$ this can be simplified to

$$
\mathcal{D}_{\mathrm{c}}= \begin{cases}\frac{1}{2}\left(S-S^{\prime} \delta_{\max }\right) \dot{\delta}_{\max }, & \dot{\delta}_{\max }>0 \\ 0, & \dot{\delta}_{\max } \leq 0\end{cases}
$$


The dissipated energy is the complement of the current maximum storable energy under the effective tractionseparation curve (see Figure 6).

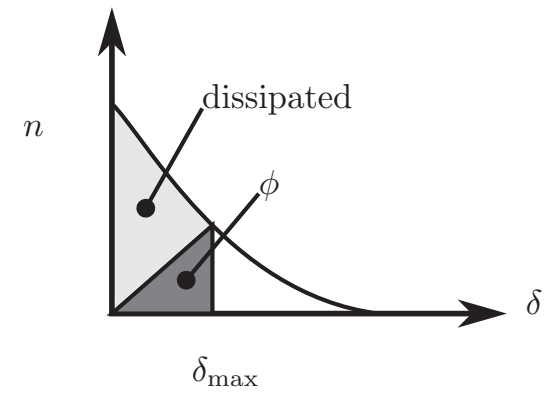

Figure 6: Effective resultant traction-separation law.

In this work, a linear strength function is used

$$
S\left(\delta_{\max }\right)=S_{0}-k \delta_{\max }
$$

where $S_{0}$ is the initial cohesive zone strength (with dimensions of force per unit length) and $k$ is the softening rate (dimensions of force per unit area). The initial strength can be specified in terms of $\sigma_{c}$, the critical stress of the material, as $S_{0}=h \sigma_{c}$. If the total work of separation per unit area of the material $\Gamma$ is specified, then $k$ follows as $k=\frac{1}{2} h \sigma_{c}^{2} / \Gamma$. The value of the effective opening displacement at the point of complete fracture is $2 \Gamma / \sigma_{c}$. From (18) the fracture dissipation rate is

$$
\mathcal{D}_{\mathrm{c}}=\frac{1}{2} S_{0} \dot{\delta}_{\max }
$$

It bears emphasis that the cohesive behavior described here is initially rigid; that is, the cohesive zones become operative upon attainment of a critical condition. In practice, the potential crack paths will be located on inter-element edges. The critical condition for cohesive zone activation is specified in terms of the resultant tractions on the edge

$$
\begin{aligned}
\mathbf{n} & :=\mathbf{n}^{\alpha} \nu_{\alpha} \\
\mathbf{m} & :=\mathbf{m}^{\alpha} \nu_{\alpha} .
\end{aligned}
$$

Note that, using (22), (23), and (20), the effective scalar traction can be written

$$
n=\sqrt{n_{N}^{2}+\left(\frac{n_{S}}{\alpha_{1}}\right)^{2}+\left(\frac{m_{N}}{\alpha_{2} h}\right)^{2}+\left(\frac{m_{S}}{\alpha_{3} h}\right)^{2}}
$$

where $n_{N}$ and $n_{S}$ are the components of the cohesive resultant traction $\mathbf{n}_{c}$ resolved along $\boldsymbol{\nu}$ and $\boldsymbol{\delta}_{S} /\left\|\boldsymbol{\delta}_{S}\right\|$, respectively (and likewise for $\mathbf{m}_{c}$ ). Consistent with (29), the critical condition determining the nucleation of new cohesive surface is

$$
f(\mathbf{n}, \mathbf{m})=\frac{n_{\mathrm{eq}}(\mathbf{n}, \mathbf{m})}{S_{0}}-1=0
$$

where $n_{\mathrm{eq}}$ is the scalar equivalent traction given by

$$
n_{\mathrm{eq}}=\left[(\mathbf{n} \cdot \boldsymbol{\nu})^{2}+\left(\frac{1}{\alpha_{1}}(\mathbf{1}-\boldsymbol{\nu} \otimes \boldsymbol{\nu}) \mathbf{n}\right)^{2}+\left(\frac{1}{\alpha_{2} h} \mathbf{m} \cdot \boldsymbol{\nu}\right)^{2}+\left(\frac{1}{\alpha_{3} h}(\mathbf{1}-\boldsymbol{\nu} \otimes \boldsymbol{\nu}) \mathbf{m}\right)^{2}\right]^{1 / 2}
$$

Through this relation, the parameters $\left\{\alpha_{1}, \alpha_{2}, \alpha_{3}\right\}$ can be interpreted as the relative strengths of the shearing and bending fracture modes relative to the in-plane opening mode. Other nucleation criteria are also possible, such as the method in [22] which ensures continuity of the tractions in time through the cohesive zone activation. 


\section{Discrete equations of motion}

The spatial discretization of the virtual power balance is done using our recently proposed discontinuous Galerkin method for shear-flexible shells [8], which we summarize. The interested reader is referred to [8] for details of the method and its implementation. We extend this method from quasi-static analysis to dynamics using a Newmark-type procedure specified in Section 4.3.

The following notation is used in this section. Let $\mathcal{T}_{h}$ be a conforming collection of isoparametric triangle elements interpolating the shell mid-surface in the reference configuration, $\mathcal{S}^{0} \simeq \mathcal{S}_{h}^{0}=\cup_{E \in \mathcal{T}_{h}} E$. By $\hat{E}$ we denote the master element in $\mathcal{A}$ that maps to $E$. The set of all edges in the triangulation is written $\Gamma=\cup_{E \in \mathcal{T}_{h}} \partial E$. The symbol $\Gamma_{I}$ signifies the set of all internal edges, $\Gamma_{I}=\Gamma \backslash \partial \mathcal{S}_{h}$.

\subsection{Interpolation of fields}

To approximate the mid-surface position and director fields, we respectively use the polynomial spaces

$$
\boldsymbol{X}_{h}=\prod_{E \in \mathcal{T}_{h}}\left[\mathcal{P}_{k}(\hat{E})\right]^{3}, \quad \quad \boldsymbol{T}_{h}=\prod\left[\mathcal{P}_{k-1}(\hat{E})\right]^{3}
$$

where $\mathcal{P}_{k}(\hat{E})$ is the set of polynomials of degree at most $k$ on $\hat{E}$. Taking the polynomial space of the positions to be one degree greater than that of the director is essential to prevent shear locking; see [8]. The discrete configuration space at any time $t \in[0, T]$ is $\mathbb{X}_{h, t}=\boldsymbol{X}_{h} \times \boldsymbol{T}_{h}$. In this work, kinematic boundary conditions are enforced strongly; consequently, the functions in $\boldsymbol{X}_{h}$ and $\boldsymbol{T}_{h}$ are required to satisfy (5).

We denote the the basis functions of $\boldsymbol{X}_{h}$ by $N^{A}, A=1,2, \ldots \mathcal{N}_{x}$, where $\mathcal{N}_{x}$ is the number of position nodes in the mesh. The mid-surface position field is approximated by

$$
\varphi_{h}\left(\xi^{\alpha}, t\right)=\sum_{A=1}^{\mathcal{N}_{x}} N^{A}\left(\xi^{\alpha}\right) \overline{\mathbf{x}}_{A}(t)
$$

where $\overline{\mathbf{x}}_{A}$ are the nodal positions. Following the Galerkin prescription, we take the set of virtual velocity fields to be identical to the actual velocities (modulo the vanishing of the virtual velocities where there are displacement boundary conditions). Thus we have

$$
\boldsymbol{\eta}\left(\xi^{\alpha}\right)=\sum_{A=1}^{\mathcal{N}_{x}} N^{A}\left(\xi^{\alpha}\right) \boldsymbol{\eta}_{A}
$$

The basis functions for the discrete director manifold $\boldsymbol{T}_{h}$ are denoted by $L^{A}, A=1,2, \ldots, \mathcal{N}_{T}$, where $\mathcal{N}_{T}$ is the number of director nodes in the mesh. The director field is interpolated according to

$$
\mathbf{t}_{h}\left(\xi^{\alpha}, t\right)=\sum_{A=1}^{\mathcal{N}_{T}} L^{A}\left(\xi^{\alpha}\right) \mathbf{t}_{A}(t)
$$

where $\mathbf{t}_{A} \in S^{2}$ are the nodal values of the director field.

The director velocity $\dot{\mathbf{t}}$ belongs to $T_{\mathbf{t}} S^{2}$, the tangent space of the director manifold, as do a number of other important quantities, including the director virtual velocity, and incremental director displacements. Approximating members of $T_{\mathbf{t}} S^{2}$ requires some care, since any $\mathbf{w} \in T_{\mathbf{t}} S^{2}$ is subject to the geometric constraint $\mathbf{w} \cdot \mathbf{t}=0$. We construct these approximations using the scheme advocated by Simo, Fox, and Rifai [23], which we summarize. We define nodal rotation tensors $\boldsymbol{\Lambda}_{A} \in S O(3)$ such that

$$
\mathbf{t}_{A}=\boldsymbol{\Lambda}_{A} \mathbf{E}_{3}, \quad A=1,2, \ldots \mathcal{N}_{T}
$$

and where the rotation axis is orthogonal to $\mathbf{E}_{3}$. Then any $\mathbf{w}_{A} \in T_{\mathbf{t}_{h}} S^{2}$ can be defined by the relation $\mathbf{w}_{A}=\boldsymbol{\Lambda}_{A} \mathbf{W}_{A}$, where $\mathbf{W}_{A}$ is a vector orthogonal to $\mathbf{E}_{3}$ (see Figure 7 ). One refers to $\mathbf{w}_{A}$ as the spatial 
representation of the field, and to $\mathbf{W}_{A}$ as the material representation. ${ }^{3}$ Clearly $\mathbf{W}_{A}$ has only two independent components; the "eliminated" component is the drilling motion. It can be shown that the drilling motion of the director expends no power [21], and hence this parameterization is ideal in the sense that it obviates Lagrange multipliers or penalty terms to control the drilling motions.

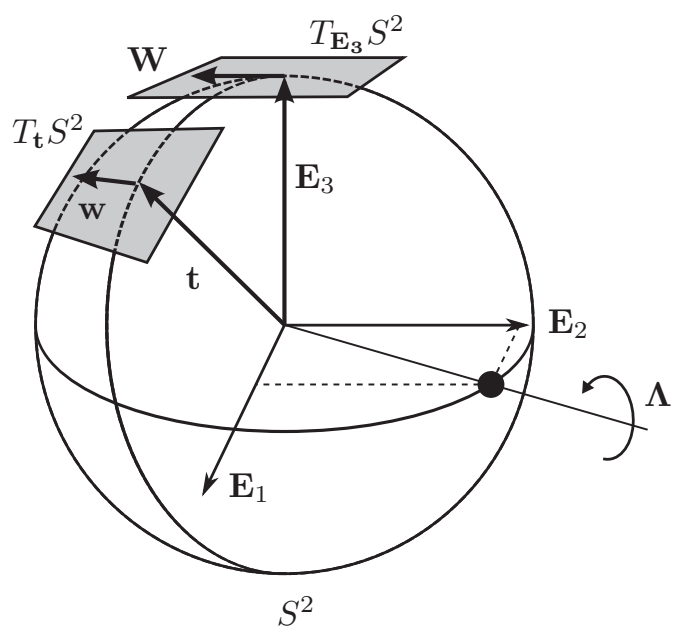

Figure 7: Parameterization of the unit sphere and its tangent space. The director $\mathbf{t}$ is the image of $\mathbf{E}_{3}$ under the rotation $\boldsymbol{\Lambda}$.

The director velocity and accelerations are approximated with the representations ${ }^{4}$

$$
\begin{aligned}
\dot{\mathbf{t}}_{h}\left(\xi^{\alpha}, t\right) & =\sum_{A=1}^{\mathcal{N}_{T}} L^{A}\left(\xi^{\alpha}\right) \dot{\mathbf{t}}_{A}(t)=\sum_{A=1}^{\mathcal{N}_{T}} L^{A}\left(\xi^{\alpha}\right) \overline{\boldsymbol{\Lambda}}_{A} \dot{\mathbf{T}}_{A}(t) \\
\ddot{\mathbf{t}}_{h}\left(\xi^{\alpha}, t\right) & =\sum_{A=1}^{\mathcal{N}_{T}} L^{A}\left(\xi^{\alpha}\right) \ddot{\mathbf{t}}_{A}(t)=\sum_{A=1}^{\mathcal{N}_{T}} L^{A}\left(\xi^{\alpha}\right) \overline{\boldsymbol{\Lambda}}_{A} \ddot{\mathbf{T}}_{A}(t)
\end{aligned}
$$

where $\overline{\boldsymbol{\Lambda}}_{A}$ is the matrix of the first two columns of $\boldsymbol{\Lambda}_{A}$, and $\dot{\mathbf{T}}_{A}, \ddot{\mathbf{T}}_{A} \in \mathbb{R}^{2}$ are nodal values for the material director velocity and acceleration. Again following Galerkin's method, the virtual director velocities are given by

$$
\mathbf{w}\left(\xi^{\alpha}\right)=\sum_{A=1}^{\mathcal{N}_{T}} L^{A}\left(\xi^{\alpha}\right) \bar{\Lambda}_{A} \mathbf{W}_{A} .
$$

The rotation tensors $\boldsymbol{\Lambda}_{A}$ evolve during the deformation. An algorithm for updating the rotation tensors is given in the Appendix.

\subsection{Spatial discretization of the equations of motion}

We discuss first the spatial discretization of the shell in the intact state; the additions to address fracture are discussed in Section 5. Inserting the discrete approximations for the kinematic fields into the the virtual

\footnotetext{
${ }^{3}$ In the following, we use the notation of lowercase boldface letters for spatial vectors, and capital boldface letters for their material counterparts in $\mathbb{R}^{2}$.

4 Strictly speaking, the interpolation of the director acceleration above is only for the tangential component. There is a centripetal component of the director acceleration as well; however, due to the constraint $\|\mathbf{t}\|=1$, this component is not independent, and is given by $-\|\dot{\mathbf{t}}\|^{2} \mathbf{t}$. It is not necessary to represent it explicitly since the director interpolation and update formula automatically satisfy the constraint.
} 
power (6), and applying the DG scheme proposed in [8], we arrive at the semi-discrete equations of motion

$$
\begin{aligned}
& \mathbf{f}_{\mathrm{x}, \mathrm{ext}}^{A}(t)-\mathbf{f}_{\mathrm{x}, \mathrm{int}}^{A}\left(\overline{\mathbf{x}}_{I}, \dot{\overline{\mathbf{x}}}_{I}\right)-\mathbf{f}_{\mathrm{x}, \mathrm{stab}}^{A}\left(\overline{\mathbf{x}}_{I}\right)=\sum_{B=1}^{\mathcal{N}_{x}} \mathbf{M}^{A B} \ddot{\mathbf{x}}_{B} \\
& \mathbf{f}_{\mathrm{T}, \mathrm{ext}}^{A}(t)-\mathbf{f}_{\mathrm{T}, \mathrm{int}}^{A}\left(\mathbf{t}_{I}, \dot{\mathbf{t}}_{I}\right)-\mathbf{f}_{\mathrm{T}, \mathrm{stab}}^{A}\left(\mathbf{t}_{I}\right)=\sum_{B=1}^{\mathcal{N}_{T}} \mathbf{I}^{A B} \ddot{\mathbf{T}}_{B}
\end{aligned}
$$

where $\mathbf{f}_{\mathrm{x}, \text { int }}^{A}$ are the internal forces on the position nodal points, $\mathbf{f}_{\mathrm{T}, \text { int }}^{A}$ are the internal moments on the director nodal points, and $\mathbf{f}_{\mathrm{x}, \mathrm{stab}}^{A}\left(\overline{\mathbf{x}}_{I}\right)$ and $\mathbf{f}_{\mathrm{T}, \mathrm{stab}}^{A}\left(\mathbf{t}_{I}\right)$ are forces associated with numerical stabilization.

The internal forces are generated from the stress power according to

$$
\begin{aligned}
\delta \mathcal{P}_{\mathrm{s}}\left(\boldsymbol{\varphi}_{h}, \mathbf{t}_{h} ; \boldsymbol{\eta}, \mathbf{w}\right) & =\int_{\mathcal{S}_{h}^{0}}\left(\mathbf{n}^{\alpha} \cdot \partial_{\alpha}^{\mathrm{DG}} \boldsymbol{\eta}+\mathbf{l} \cdot \mathbf{w}+\mathbf{m}^{\alpha} \cdot \partial_{\alpha}^{\mathrm{DG}} \mathbf{w}\right) d \mathcal{S}^{0} \\
& =\sum_{A \in \mathcal{N}_{x}} \mathbf{f}_{\mathrm{x}, \mathrm{int}}^{A} \cdot \boldsymbol{\eta}_{A}+\sum_{A \in \mathcal{N}_{T}} \mathbf{f}_{\mathrm{T}, \mathrm{int}}^{A} \cdot \mathbf{W}_{A},
\end{aligned}
$$

where $\partial_{\alpha}^{\text {DG }}$ are so-called $D G$ derivatives, which are projections of the derivative operators onto the discontinuous piecewise polynomial spaces, necessary to maintain consistency [24, 25]. For the full derivation and the explicit form of the internal nodal forces the reader is again referred to [8]. The stress resultants $\left\{\mathbf{n}^{\alpha}, \mathbf{m}^{\alpha}, \mathbf{l}\right\}$ are given by constitutive functions, usually written in terms of $2 \mathrm{D}$ surface tensors and internal variables; see [21].

As in most discontinuous Galerkin methods, stabilization is needed to remove zero energy modes introduced by the nonconforming approximations. We use the penalty terms

$$
\delta \mathcal{P}_{\text {stab }}(\boldsymbol{\varphi}, \mathbf{t} ; \boldsymbol{\eta}, \mathbf{w})=\int_{\Gamma_{I}}\left(\frac{\beta_{x}}{h^{e}} \llbracket \boldsymbol{\varphi} \rrbracket \cdot \llbracket \boldsymbol{\omega} \rrbracket+\frac{\beta_{T}}{h^{e}} \llbracket \mathbf{t} \rrbracket \cdot \llbracket \mathbf{w} \rrbracket\right) \bar{j}^{0} d s,
$$

where $\llbracket \cdot \rrbracket$ is the jump operator defined in Eq. (11). The symbol $h^{e}$ is a local measure of the mesh size, here taken as the length of the edge $e \in \Gamma_{I}$. In the present work, the penalty parameters are normalized by taking $\beta_{x}=c_{x} E h$ and $\beta_{T}=c_{T} E h^{3} / 12$, where $h$ is the shell thickness (and is not to be confused with the mesh size $h_{e}$ ), $E$ is the Young's modulus of the material, and $c_{x}, c_{T}$ are (problem dependent) positive real numbers taken sufficiently large. Substitution of the interpolations into (42) gives the stabilization nodal forces as

$$
\begin{aligned}
\mathbf{f}_{\mathrm{x}, \mathrm{stab}}^{A} & = \pm \sum_{e \in \Gamma_{I}} \int_{e} \frac{\beta_{x}}{h^{e}} \llbracket \boldsymbol{\varphi}_{h} \rrbracket N^{A} \bar{j}^{0} d s, \\
\mathbf{f}_{\mathrm{T}, \mathrm{stab}}^{A} & = \pm \sum_{e \in \Gamma_{I}} \int_{e} \frac{\beta_{T}}{h^{e}}\left(\overline{\boldsymbol{\Lambda}}^{A}\right)^{\top} \llbracket \mathbf{t}_{h} \rrbracket L^{A} \bar{j}^{0} d s
\end{aligned}
$$

The stabilization forces are computed with one-dimensional interface elements (Figure 8). The stabilization terms have a nearly identical form to the cohesive zone virtual internal power, and thus the interface elements provide a convenient means to compute the cohesive zone forces if a crack nucleates on the edge as explained in Section 5.

The virtual kinetic energy rate gives rise to the dynamic terms

$$
\begin{aligned}
\delta \dot{\mathcal{K}} & =\int_{\mathcal{S}_{h}^{0}}\left(\bar{\rho}^{0} \ddot{\varphi}_{h} \cdot \boldsymbol{\eta}+\bar{I}^{0} \ddot{\mathbf{t}}_{h} \cdot \mathbf{w}\right) d \mathcal{S}^{0} \\
& =\sum_{A=1}^{\mathcal{N}_{x}} \sum_{B=1}^{\mathcal{N}_{x}} \boldsymbol{\eta}_{B} \cdot \mathbf{M}^{A B} \ddot{\overline{\mathbf{x}}}+\sum_{A=1}^{\mathcal{N}_{T}} \sum_{B=1}^{\mathcal{N}_{T}} \mathbf{W}_{A} \cdot \mathbf{I}^{A B} \ddot{\mathbf{T}}_{B}
\end{aligned}
$$




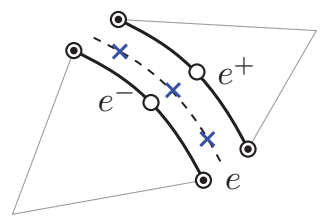

- Mid-surface node

- Director node

$\times$ Quadrature point

Figure 8: Schematic of the interface element used to compute the nodal forces due to the stabilization terms, with the polynomial degree of the shape functions $k=2$. The edges $e^{-}$and $e^{+}$coincide in the reference configuration. The interface element is reused to compute the cohesive tractions if the fracture criterion is satisfied on the interface, as described in Section 5 .

in which $\mathbf{M}^{A B}$ and $\mathbf{I}^{A B}$ are the consistent mass matrices given by

$$
\begin{aligned}
\mathbf{M}^{A B} & =\int_{\mathcal{S}_{h}^{0}} \bar{\rho}^{0} N^{A} N^{B} \mathbf{1}_{3} \bar{j}^{0} d \mathcal{A} \\
\mathbf{I}^{A B} & =\int_{\mathcal{S}_{h}^{0}} \bar{I}^{0} L^{A} L^{B}\left(\overline{\boldsymbol{\Lambda}}_{A}\right)^{\top} \overline{\boldsymbol{\Lambda}}_{B} \bar{j}^{0} d \mathcal{A}
\end{aligned}
$$

In the preceding, $\mathbf{1}_{3}$ represents the identity tensor in $\mathbb{R}^{3}$. Note that $\mathbf{I}^{A B}$ is dependent on the current configuration through the nodal rotation matrix $\boldsymbol{\Lambda}_{A}$.

\subsection{Newmark time integration scheme}

Specialized numerical integration algorithms of the equations of motion for directed surfaces has been investigated by a number of authors, see e.g., [26-28]. These schemes have been designed with various goals in mind, such as exactly preserving certain integrals of the motion. For the sake of ease of implementation, we propose a simple method that extends the popular explicit version of Newmark's method to shells, while still preserving the geometric constraints of the director field.

The mid-surface positions may be integrated with the standard explicit Newmark formulae; details of this scheme are widely available (see, e.g., [29]). We focus our discussion on the non-standard director field. A standard incremental integration procedure is envisioned whereby the nodal kinematics are advanced forward from the initial conditions through a sequence of discrete times $\left\{t^{n}\right\}_{n=0}^{N}$ partitioning the interval $(0, T)$. Here and in the remainder of this section, the time level of nodal quantities is indicated by a superscript. By $\Delta t$ we indicate the increment in time between successive time levels (not necessarily constant throughout the procedure).

To integrate the director dynamics, we apply the Newmark formulae to the material director increments, velocities, and accelerations. We introduce the nodal material director increments $\Delta \mathbf{T}_{A}, A=1,2, \ldots, \mathcal{N}_{T}$. The Newmark formulae for the material director increment and the material director velocity predictor are

$$
\begin{gathered}
\Delta \mathbf{T}_{B}=\Delta t \dot{\mathbf{T}}_{B}^{n}+\frac{\Delta t^{2}}{2} \ddot{\mathbf{T}}_{B}^{n} \\
\left(\dot{\mathbf{T}}_{B}^{n+1}\right)^{\text {pre }}=\dot{\mathbf{T}}_{B}^{n}+(1-\gamma) \Delta t \dot{\mathbf{T}}_{B}^{n}
\end{gathered}
$$

where the algorithmic parameter $\gamma \in[0,1]$ allows one to control the amount of numerical damping. Second order accuracy is achieved if and only if the value of $\gamma=\frac{1}{2}$ is taken [29], leading to the central difference method.

At this point, the geometrically exact kinematic update algorithm described in [23] is applied to get $\left(\mathbf{t}_{B}^{n}, \Delta \mathbf{T}_{B}\right) \mapsto \mathbf{t}_{B}^{n+1}$. This algorithm also provides the update $\left(\boldsymbol{\Lambda}_{B}^{n}, \Delta \mathbf{T}_{B}\right) \mapsto \boldsymbol{\Lambda}_{B}^{n+1}$ for the nodal rotation matrices. The kinematic update algorithm makes use of the exponential map, which ensures that the nodal director values remain in $S^{2}$. This update has an explicit form that is given in the Appendix. It bears emphasis that the nodal increments are not summed with the current values, as the topology of the director field is not a linear space. 
The material velocity predictor is transformed to its spatial counterpart by $\left(\dot{\mathbf{t}}_{B}^{n+1}\right)^{\text {pre }}=\Lambda_{B}^{n}\left(\dot{\mathbf{T}}_{B}^{n+1}\right)^{\text {pre }}$. The material accelerations are solved for using the equations of motion

$$
\sum_{B=1}^{\mathcal{N}_{T}} \mathbf{I}^{A B} \ddot{\mathbf{T}}_{B}=\mathbf{f}_{\mathrm{T}, \text { ext }}^{A}\left(t^{n+1}\right)-\mathbf{f}_{\mathrm{T}, \text { int }}^{A}\left(\mathbf{t}_{B}^{n+1},\left(\dot{\mathbf{t}}_{B}^{n+1}\right)^{\mathrm{pre}}\right)-\mathbf{f}_{\mathrm{T}, \mathrm{stab}}^{A}\left(\mathbf{t}_{B}^{n+1}\right)
$$

The final step is the material velocity corrector

$$
\dot{\mathbf{T}}_{A}^{n+1}=\left(\dot{\mathbf{T}}_{A}^{n+1}\right)^{\mathrm{pre}}+(1-\gamma) \Delta t \ddot{\mathbf{T}}_{A}^{n+1}
$$

In order to ensure stability of the explicit time integration operator, we choose the size of $\Delta t$ through an element by element estimate (see, e.g., [29]) using the shear and longitudinal elastic wave speeds, modified to account for the DG discretization according to the method in [30]. In most practical cases, the flexural waves are much slower and can be ignored in the time step estimation.

In our implementation, we have elected to lump the mass matrices (using the scheme in [31]) to avoid the solution of a linear system in the calculation of the accelerations. Alternatively, one may take advantage of the block diagonal structure of the consistent mass matrices to compute the accelerations efficiently element by element. This special structure is due to the fact that each discontinuous Galerkin shape function has support over only a single element.

It is important to note that the dependence of the rotary inertia matrix $\mathbf{I}^{A B}$ on the current configuration (through the rotation matrices $\boldsymbol{\Lambda}_{A}$ ) implies that it must be re-assembled as the shell deforms. In our implementation, the rotary inertia matrix is recomputed at every time step. The computational expense of this re-assembly has not been systematically investigated, but is expected to be small relative to the computation of the internal forces. Should it later prove to be burdensome in some application, re-assembly can be undertaken periodically, as the changes in the director field between successive time steps are small due to the time step restrictions imposed by stability. A mitigating factor of the rotary inertia re-assembly is that it is a trivially parallelizable computation, requiring no communication, thanks again to the elementwise support of the DG shape functions.

The proposed method is amenable to parallelization through a standard domain decomposition approach and message passing. In our implementation, the domain partitioning is performed using the METIS library [32] to minimize the number of boundary elements and hence the communication. Communication of forces on processor boundaries is handled through ghost elements; each processor sub-domain includes copies of elements one layer deep into adjacent sub-domains. The interface forces (from the stabilization terms or the cohesive zones) on the inter-processor boundaries are handled by creating an interface element on only one of the partitions. The interface element is placed between the "real" shell element on the boundary and the ghost element that represents a shell element in the neighbor processor. This has the potential to introduce an unbalance in the processor loads, but in practice does not cause much harm as the shell elements on a given processor domain significantly outnumber the boundary interface elements unless the number of elements per processor is very small.

\section{Cohesive zone implementation}

Activation of a cohesive zone on an element edge is determined by the resultant tractions acting on the edge (cf. Eqs. (30) and (31)). The resultants can be estimated at the quadrature points of the interface elements by directly applying the constitutive law to the interpolated generalized strains, or by $L^{2}$ projection of the resultants already computed at the bulk shell element quadrature points (from the internal force calculation). This second option is more efficient for complex material behaviors requiring a local nonlinear solution procedure, e.g., metal plasticity. Since equilibrium is enforced weakly, tractions predicted by neighboring bulk elements are not precisely equal at the interface. Unique values are assigned by using the average trace operator, so that we take

$$
\left.\mathbf{n}\right|_{e}=\left.\{\mathbf{n}\} \quad \mathbf{m}\right|_{e}=\{\mathbf{m}\}
$$


as the values on edge $e$ to compute $n_{\text {eq }}$. The nucleation criterion (30) is averaged spatially over the edge according to

$$
\bar{f}=\frac{1}{|e|} \int_{e} f\left(\left.\mathbf{n}\right|_{e},\left.\mathbf{m}\right|_{e}\right) \bar{j}^{0} d s
$$

The cohesive zone is activated on the edge if $\bar{f} \geq 0$. The criterion is evaluated on every edge, at each time step. For simplicity, we do not address re-contact of cohesive surfaces or crack flanks under compression in this work.

\subsection{Activation procedures}

An important feature of the proposed method is that a cohesive zone can nucleate on any edge in the mesh without requiring the introduction of new degrees of freedom, and therefore no associated data structures need be modified. This is the key to the scalability of the method. This condition is due to the special property of the DG basis functions that each has support over only a single element. Additionally, this advantage is achieved without sacrificing consistency of the method, since compatibility of the kinematic fields is enforced weakly by the DG derivatives and stabilization terms.

To nucleate a cohesive zone, the enforcement of compatibility must be released. It is important to note that the interpolation of the DG derivative of the kinematic fields at quadrature points in the shell elements involves degrees of freedom on adjacent faces of neighboring elements, see Fig. 9. The cohesive zone activation on an internal edge is thus a two-step process:

1. Switch off the neighbor contributions from the newly fractured edge in the DG derivative in both neighboring shell elements.

2. Cease computing the stabilization forces on the interface element, and start computing the nodal forces determined by the traction-separation law.

The first step is shown schematically in Figure 9. Denote by $\partial E_{\text {intact }}$ the set of all edges of element $E$ where no cohesive fracture has yet nucleated. In the center grey element, the degrees of freedom on $\partial E_{\text {intact }}$ (as well as on the element itself) are used in the interpolation of the DG derivative inside the element. Initially the set $\partial E_{\text {intact }}$ contains all 3 edges of the triangular element (image on left hand side). When the cohesive zone activates on edge $\hat{e}$, it is removed from $\partial E_{\text {intact }}$ (image on right hand side). This process must be performed reciprocally on both shell elements adjacent to the newly activated cohesive zone edge. To aid this process, a data structure is created at the start of the simulation that encodes the map

$$
P: e \rightarrow\left(E_{\text {left }}, \hat{e}_{\text {left }}\right) \times\left(E_{\text {right }}, \hat{e}_{\text {right }}\right)
$$

$P$ maps each interface element in the mesh $e$ to the adjacent shell elements $E_{\text {left }}$ and $E_{\text {right }}$, and also gives the local number of the edge inside the element, $\hat{e}_{\text {left }}, \hat{e}_{\text {right }} \in\{1,2,3\}$. When an intact edge reaches the critical condition for cohesive zone activation, the map $P$ is utilized to switch off the edge contributions to the DG derivative in the adjacent shell elements.

In parallel computation, an additional step must be taken if the cohesive zone is activating at a processor boundary. In this case, the fracture will be detected on an interface element between a real element and a ghost element. For each interface element on a processor boundary, an additional communication is made along with the nodal forces, consisting of a Boolean variable indicating whether the interface is intact. These additional data are much smaller than the nodal forces already being communicated; consequently, the scalability of the method is not harmed.

The second step of the cohesive zone activation introduces the traction-separation law describing the fracture process. The interface elements responsible for enforcing the stabilization terms in the pre-fractured state are recycled to host the traction-separation law terms. Defining the stabilization resultant tractions

$$
\mathbf{n}_{\text {stab }}\left(\llbracket \boldsymbol{\varphi}_{h} \rrbracket\right):=\frac{\beta_{x}}{h^{e}} \llbracket \varphi_{h} \rrbracket \quad \mathbf{m}_{\mathrm{stab}}\left(\llbracket \mathbf{t}_{h} \rrbracket\right):=\frac{\beta_{T}}{h^{e}} \llbracket \mathbf{t}_{h} \rrbracket
$$



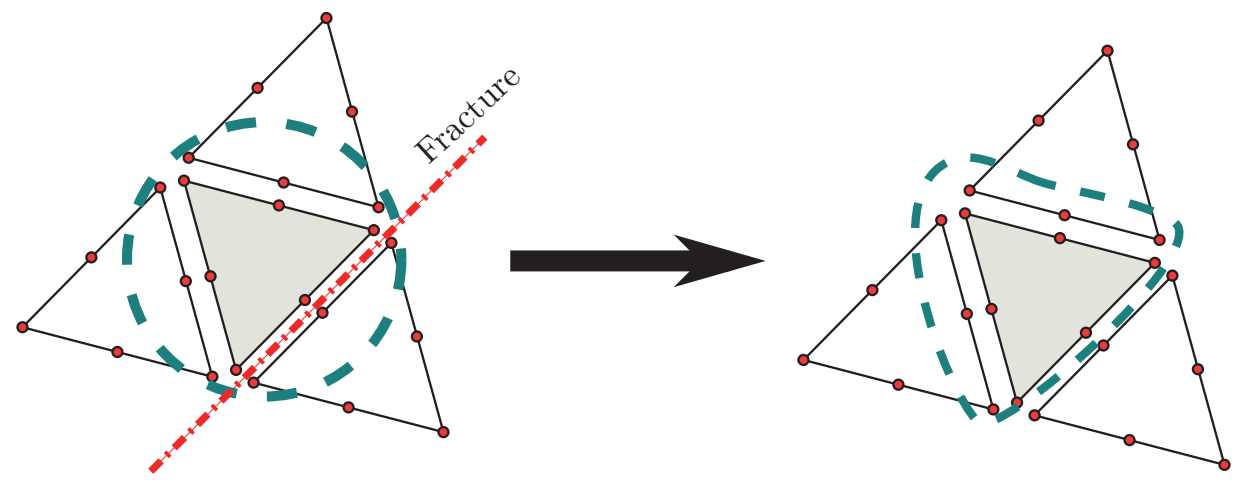

Figure 9: The DG derivative weakly enforces compatibility of the strains across element edges. To introduce fracture on the edge indicated on the left, the DG derivative in the grey shell element must be modified to omit the cracked edge, as in the image on the right. The operation must also be performed reciprocally on the other shell element adjacent to the fracture.

the DG stabilization in the virtual power balance can be rewritten in the form

$$
\delta \mathcal{P}_{\text {stab }}=\sum_{e \in \Gamma_{I}} \int_{e}\left(\mathbf{n}_{\text {stab }}\left(\llbracket \boldsymbol{\varphi}_{h} \rrbracket\right) \cdot \llbracket \boldsymbol{\eta} \rrbracket+\mathbf{m}_{\text {stab }}\left(\llbracket \mathbf{t}_{h} \rrbracket\right) \cdot \llbracket \mathbf{w} \rrbracket\right) \bar{j}^{0} d s
$$

Let $\Gamma_{c}$ be the set of all active cohesive zone edges. The virtual power of the cohesive zones is

$$
\delta \mathcal{P}_{\mathrm{c}}=\sum_{e \in \Gamma_{c}} \int_{e}\left(\mathbf{n}_{\mathrm{c}}\left(\llbracket \boldsymbol{\varphi}_{h} \rrbracket, \llbracket \mathbf{t}_{h} \rrbracket,\left\{z_{A}\right\}\right) \cdot \llbracket \boldsymbol{\eta} \rrbracket+\mathbf{m}_{\mathrm{c}}\left(\llbracket \boldsymbol{\varphi}_{h} \rrbracket, \llbracket \mathbf{t}_{h} \rrbracket,\left\{z_{A}\right\}\right) \cdot \llbracket \mathbf{w} \rrbracket\right) \bar{j}^{0} d s
$$

In this form, it is easy to see that the nodal forces due to the cohesive zone may be computed simply by changing the functional form of the constitutive law on the interface element:

$$
\begin{aligned}
\mathbf{n}_{\text {stab }}\left(\llbracket \boldsymbol{\varphi}_{h} \rrbracket\right) & \mapsto \mathbf{n}_{\mathrm{c}}\left(\llbracket \boldsymbol{\varphi}_{h} \rrbracket, \llbracket \mathbf{t}_{h} \rrbracket, \llbracket \dot{\boldsymbol{\varphi}}_{h} \rrbracket, \llbracket \dot{\mathbf{t}}_{h} \rrbracket,\left\{z_{A}\right\}\right) \\
\mathbf{m}_{\text {stab }}\left(\llbracket \boldsymbol{\varphi}_{h} \rrbracket\right) & \mapsto \mathbf{m}_{\mathrm{c}}\left(\llbracket \boldsymbol{\varphi}_{h} \rrbracket, \llbracket \mathbf{t}_{h} \rrbracket, \llbracket \dot{\boldsymbol{\varphi}}_{h} \rrbracket, \llbracket \dot{\mathbf{t}}_{h} \rrbracket,\left\{z_{A}\right\}\right)
\end{aligned}
$$

For completeness, the nodal forces on the interface element are given by

$$
\begin{aligned}
\mathbf{f}_{\mathrm{x}, \mathrm{c}}^{A} & = \pm \sum_{e \in \Gamma_{c}} \int_{e} \mathbf{n}_{\mathrm{c}} N^{A} \bar{j}^{0} d s \\
\mathbf{f}_{\mathrm{T}, \mathrm{c}}^{A} & = \pm \sum_{e \in \Gamma_{c}} \int_{e}\left(\boldsymbol{\Lambda}_{A}\right)^{\top} \mathbf{m}_{\mathrm{c}} L^{A} \bar{j}^{0} d s
\end{aligned}
$$

which are summed into the internal force array.

\section{Applications}

In each of the applications that follow, we take the Newmark $\gamma$-parameter to be $1 / 2$ (i.e., the central difference method). We use $k=2$ for the shape functions, i.e., quadratic displacements and linear director fields. For the shell bulk constitutive behavior, we use a resultant-based plasticity model as described in $[33,34]$. It bears noting that the use of the resultant based model, along with the resultant-based cohesive laws proposed in Section 3, means that no numerical integration through the shell thickness is required. The material behavior is taken to be homogeneous and isotropic, and linear isotropic hardening is used. In addition to the elastic modulus $E$ and Poisson's ratio $\nu$ describing the elastic behavior, the parameters of the model are the initial yield strength $Y_{0}$, the isotropic hardening modulus $H$, and the shear correction factor $\kappa$, which we have taken as $5 / 6$ in all cases. The mass density in the undeformed configuration is written as $\rho^{0}$. In order to simplify the constitutive updates, surface tensors are computed in local Cartesian bases according to the method in Appendix $\mathrm{C}$ of [23]. 


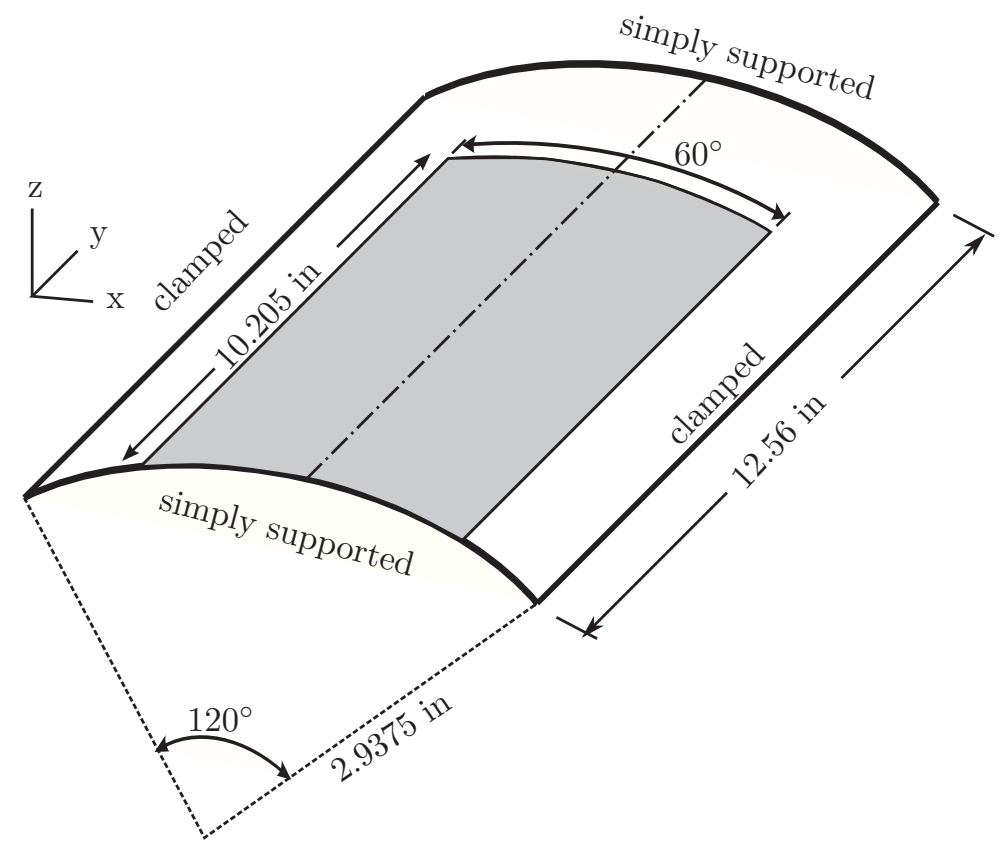

Figure 10: Schematic of the impulsively loaded cylinder problem. An initial velocity of $5650 \mathrm{in} / \mathrm{s}$ in the negative $z$-direction is applied to the grey region.

\subsection{Impulsively loaded elastic-perfectly plastic cylindrical panel}

This example tests the ability of the element formulation and the time integration procedure to simulate large deformations and inelastic material behavior. The considered scenario is a cylindrical panel subject to impulsive loading based on the experiments in [35], and has been used as a benchmark for shell elements in the literature. The panel is composed of an elastic, perfectly plastic material, with the geometry and boundary conditions shown in Figure 10, and the parameters of the problem are specified in Table 1. An initial velocity of magnitude $V_{0}$ is applied to the region in grey in Figure 10. The output of interest is the vertical deflection of the panel at its center point over a time period of $1 \mathrm{~ms}$.

Table 1: Parameters for the impulsively loaded elastic-perfectly plastic cylindrical panel.

\begin{tabular}{cccccccc}
\hline$E$ & $\nu$ & $h$ & $\rho^{0}$ & $Y_{0}$ & $V_{0}$ & $c_{x}$ & $c_{T}$ \\
\hline $10.5 \times 10^{6} \mathrm{psi}$ & 0.33 & $0.125 \mathrm{in}$ & $2.5 \times 10^{-4} \mathrm{lb} \mathrm{s}^{2} / \mathrm{in}^{4}$ & $44 \times 10^{3} \mathrm{psi}$ & $5650 \mathrm{in} / \mathrm{s}$ & 3 & 3 \\
\hline
\end{tabular}

The mesh used for the calculation is shown in Figure 11a; symmetry is used to model only half of the shell. The mesh used in the present calculations has 16 elements in the axial direction and 6 elements in the circumferential direction. The center point deflection predictions of the present method are compared with the experimental observations, as well as with predictions from the Belytschko-Tsay stabilized one point quadrature shell element [36]. The displacement histories predicted by both methods are shown in Figure 11c, along with the experimental results for reference. The proposed method is shown to achieve excellent agreement with experiments.

\subsection{Mode III tearing of a plate}

The plate tearing experiments presented in [37, 38] are used to illustrate that the cohesive zone model is capable of simulating out-of-plane shear failure. In this scenario, a notched plate is loaded in a nominally mode III configuration as follows: the halves of the plate above and below the notch are clamped and displaced in opposing senses orthogonally to the plate surface (see Figure 12). In order to save computational 

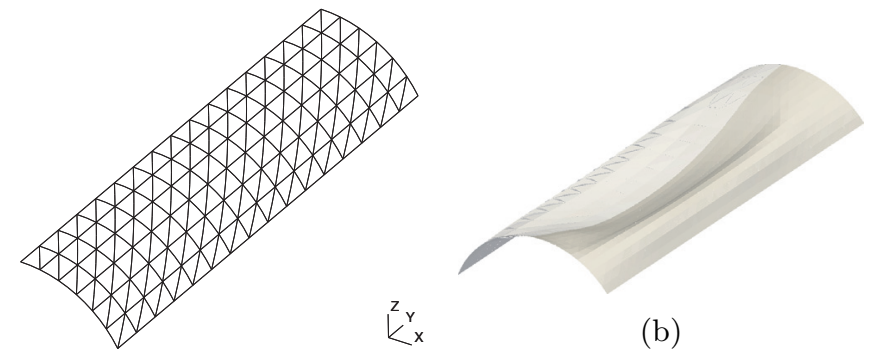

(a)

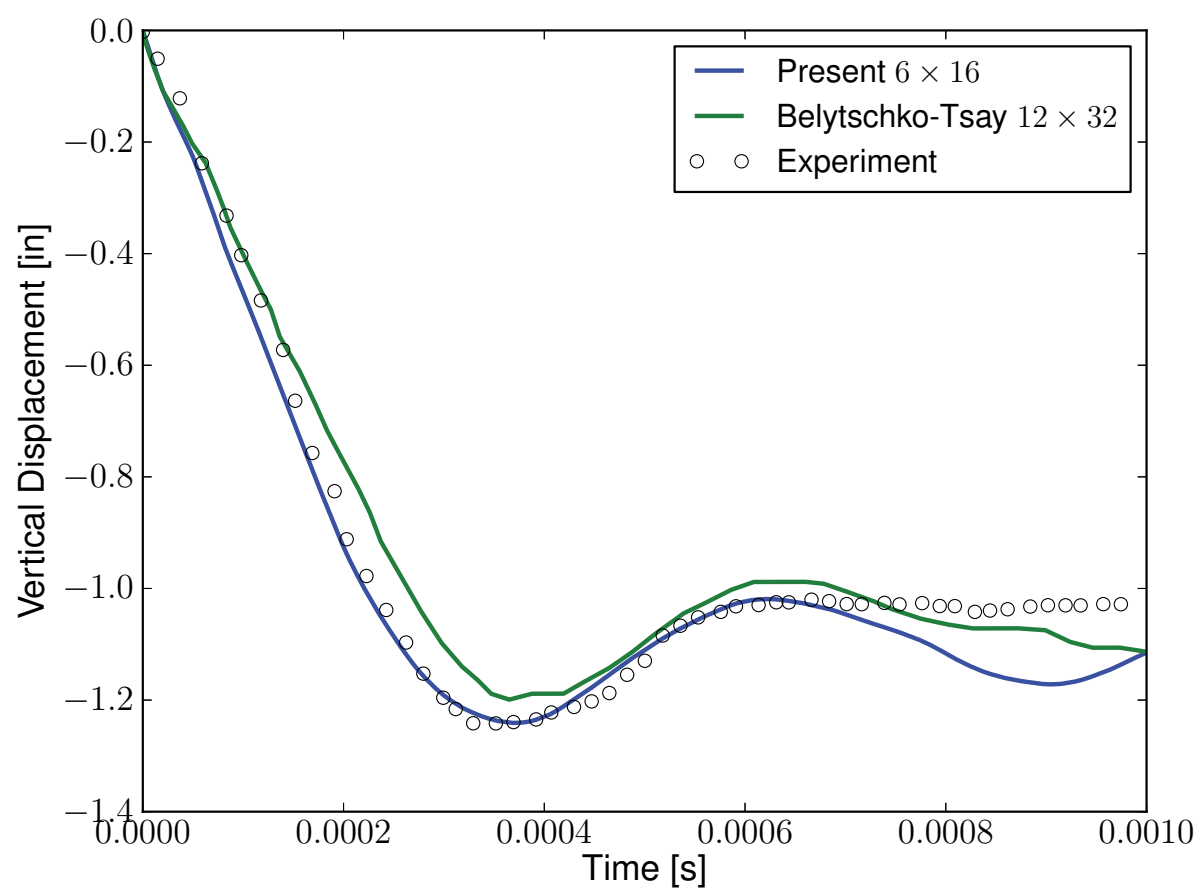

(c)

Figure 11: (a) Mesh used for the impulsively loaded cylindrical panel problem, showing symmetry condition. (b) Deformed shape of the panel at the end of the simulation. (Numerical results have been reflected to show the entire shell.) (c) Experimental and numerical measurements of the history of the vertical displacement of the center point of the panel. The density of the employed mesh is indicated in the legend for both numerical methods. 
effort, the parts of the plate fixed within the clamps, shown in Figure 12 as the region within the dashed lines, is not meshed. The mesh is aligned to contain the expected crack propagation direction. The boundary conditions are imposed as velocities on the edge of the clamped region, with the transverse velocity set as $v_{z}= \pm V_{0}$ and all other degrees of freedom on the clamped edge set to zero. The experiments were conducted under quasi-static conditions, with cross-head velocities of $4 \times 10^{-5} \mathrm{~m} / \mathrm{s}$. The numerical simulations were performed with explicit dynamics; in order to speed up the computations, the loading rate was scaled up by a factor of $10^{5}$, so that $V_{0}=4 \mathrm{~m} / \mathrm{s}$. The velocity boundary condition was ramped up smoothly from zero to $V_{0}$ over a short time duration to suppress transient elastic oscillations.

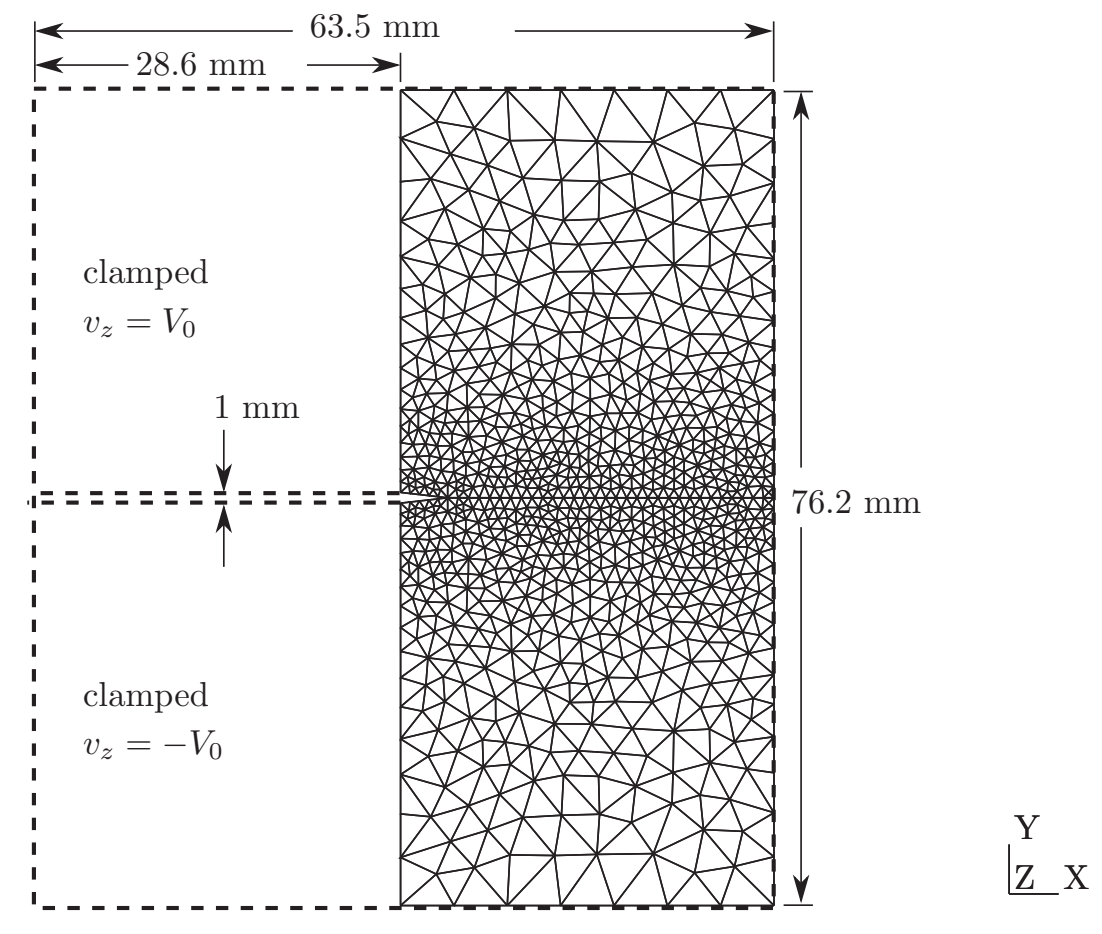

Figure 12: Geometry, mesh, and boundary conditions for mode III tearing simulation.

The plate material is 6061-T6 aluminum alloy. The elastic-plastic material properties for this alloy used in the numerical simulations are given in Table 2. A value of $\sigma_{c}=325 \mathrm{MPa}$ for the cohesive strength was assumed. The value of the work of separation was varied as a parameter to demonstrate its influence on the predictions; we took $\Gamma$ to be $\left\{0.5 Y_{0} h, 0.75 Y_{0} h, 1.0 Y_{0} h\right\}$.

The computed vertical force $F$ vs. imposed displacement $\Delta$ is shown in dimensionless form in Figure 13 , alongside the experimental results. ${ }^{5}$ It can be seen in the plots that the simulations capture the steadystate response with reasonable agreement. The match in the force initial peak could be improved by using traction-separation laws that are specialized for ductile fracture in plates and shells (e.g. [16-20]). Another source of discrepancy may come from the assumption that the clamping is perfectly rigid; the authors in [38] report that modeling the compliance of the test device fixture was necessary to obtain good agreement when using their ductile fracture model. However, these steps are beyond the scope of the current paper.

Figure 15 shows snapshots of the evolution of the deformation of the plate and the propagation of the mode III crack. The colors represent the distribution of accumulated plastic strain. It is thus clear that the framework is able to represent the through-thickness shearing mode that is unavailable in Kirchhoff-Love shell formulations. For comparison, Figure 14 shows photographs of the final deformed configuration of the plate at the end of the experiment.

\footnotetext{
${ }^{5}$ The quantity $b$ in the normalization is the original ligament length, $b=33.1 \mathrm{~mm}$
} 
Table 2: Parameters used in the mode III plate tearing simulation.

\begin{tabular}{cccccccccccc}
\hline$E$ & $\nu$ & $h$ & $\rho^{0}$ & $Y_{0}$ & $H$ & $\sigma_{c}$ & $\alpha_{1}$ & $\alpha_{2}$ & $\alpha_{3}$ & $c_{x}$ & $c_{T}$ \\
\hline $72 \mathrm{GPa}$ & 0.343 & $2 \mathrm{~mm}$ & $2700 \mathrm{~kg} / \mathrm{m}^{3}$ & $320 \mathrm{MPa}$ & $894 \mathrm{MPa}$ & $325 \mathrm{MPa}$ & 1 & 1 & 1 & 3 & 3 \\
\hline
\end{tabular}

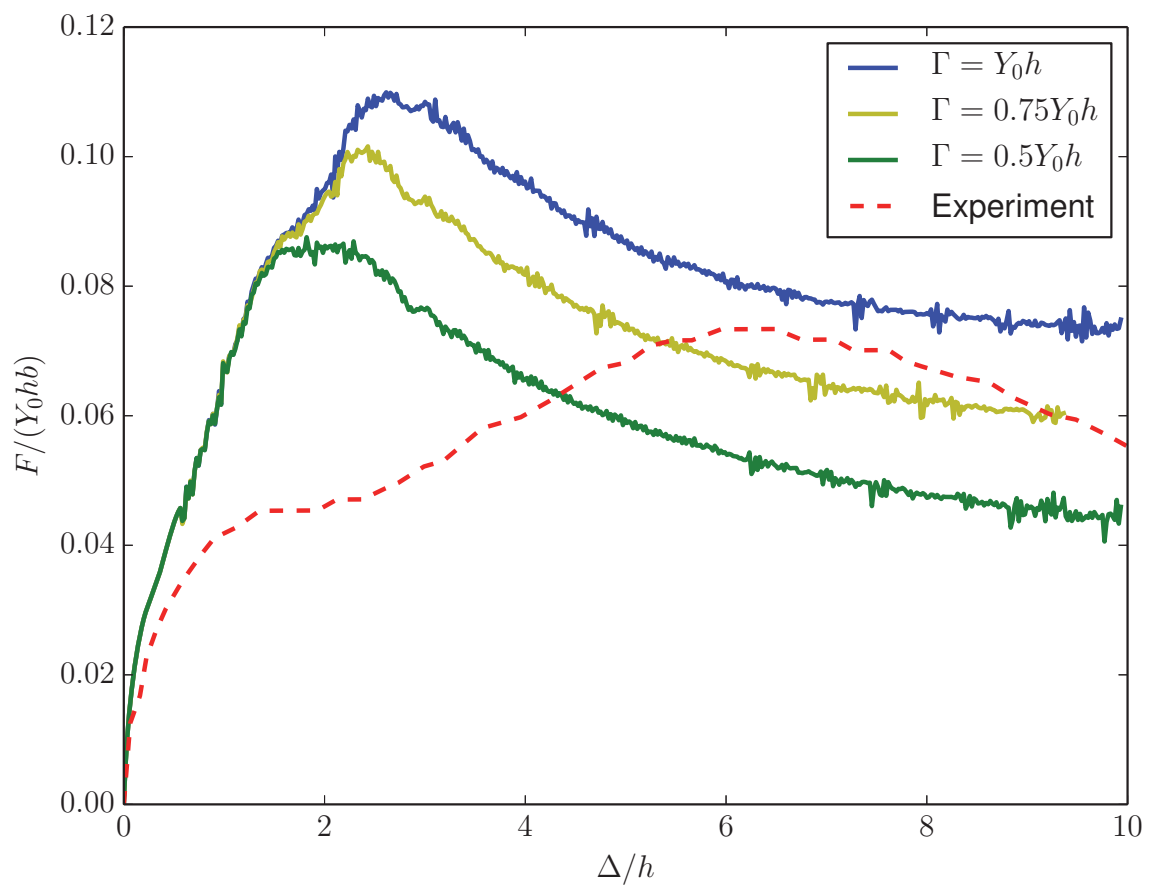

Figure 13: Vertical force vs. imposed vertical displacement at the clamp for the mode III plate tearing simulation. 

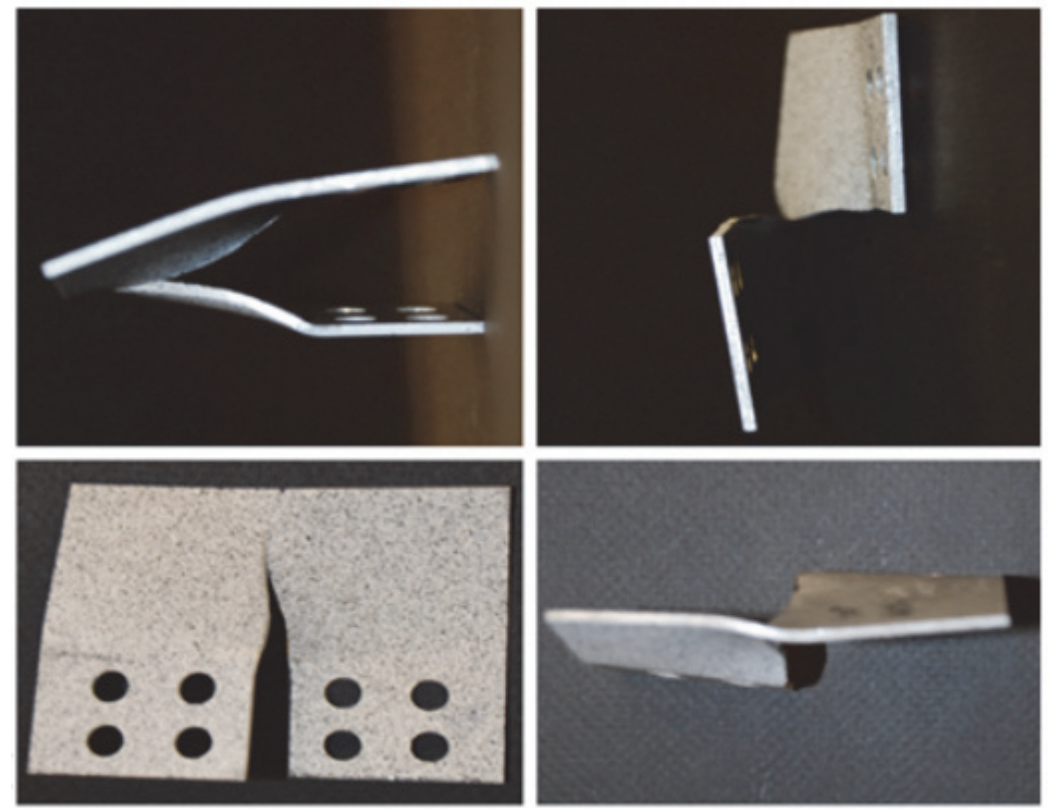

Figure 14: Photographs of specimen from the mode III plate tearing experiments (Image source: Li et al. [38]).

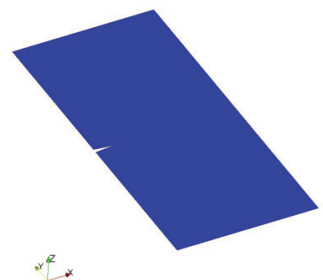

(a) $\Delta / h=0$

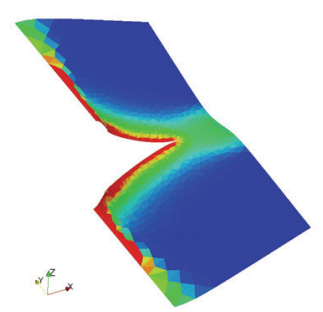

(d) $\Delta / h=6$

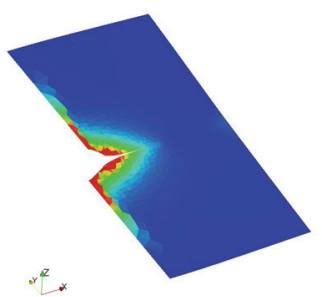

(b) $\Delta / h=2$

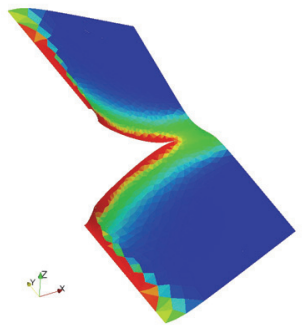

(e) $\Delta / h=8$

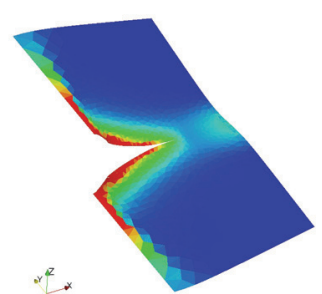

(c) $\Delta / h=4$

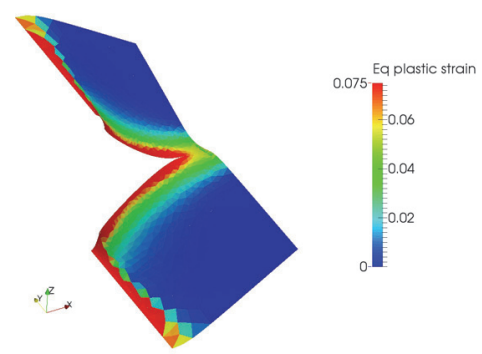

(f) $\Delta / h=10$

Figure 15: Deformation of mode III tearing simulation for the case $\Gamma=0.5 Y_{0} h$. The contoured quantity is the hardening variable of the stress resultant plasticity law, which may be regarded as the equivalent plastic strain. Note that by (c), the plastic zone has extended across the ligament. 
Table 3: Parameters for the simulation for the beam blast experiments.

\begin{tabular}{|c|c|c|c|c|c|c|c|c|c|}
\hline$E$ & $\nu$ & $h$ & \multicolumn{3}{|c|}{$\rho^{0}$} & \multicolumn{2}{|c|}{$Y_{0}$} & $H$ & $\sigma_{c}$ \\
\hline \multirow[t]{3}{*}{$68.9 \mathrm{GPa}$} & 0.343 & $6.53 \mathrm{~mm}$ & \multicolumn{3}{|c|}{$2693 \mathrm{~kg} / \mathrm{m}^{3}$} & \multicolumn{2}{|c|}{$275 \mathrm{MPa}$} & $1.33 \mathrm{GPa}$ & $375 \mathrm{MPa}$ \\
\hline & & $\Gamma$ & $\alpha_{1}$ & $\alpha_{2}$ & $\alpha_{3}$ & $c_{x}$ & $c_{T}$ & & \\
\hline & & 0 & 1 & 1 & 1 & 3 & 3 & & \\
\hline
\end{tabular}

\subsection{Beam blast experiments of Menkes and Opat}

This example, referenced in the Introduction, demonstrates the increased importance of through-thickness shearing on fracture as the loading rate increases. This application comes from the experiments of Menkes and Opat [15], in which planar explosive charges were affixed to the top face of a clamped beam and detonated; the impulse delivered to the beam was controlled by varying the thickness of the charge. The beams are made of aluminum alloy 6061-T6; typical elastic-plastic material properties of this alloy are assumed, shown in Table 3. The beam is $200 \mathrm{~mm}$ in span, $6 \mathrm{~mm}$ in depth, and $25 \mathrm{~mm}$ in width. We model the impulsive load approximately by applying a spatially uniform initial velocity in the downward direction to the entire beam. The cohesive strength is taken as $375 \mathrm{MPa}$. The work of separation is of secondary importance in this experiment, as it affects the residual velocity of the beam after fracture, but not the crack initiation, which is the focus of this example. For simplicity, we thus take the work of separation to be zero.

The post-mortem photographs of the experiments in Figure 1 show that the observed response can be divided into three regimes, arranged in order of increasing impulse:

1. Permanent deflection due to bending and stretching without fracture;

2. Necking and tensile failure at the supports after large deflection;

3. Shear-off at the supports with little permanent deformation.

Three particular levels of impulse were simulated: $10.9 \mathrm{kPa} \mathrm{s}, 17.8 \mathrm{kPa} \mathrm{s}, 39.6 \mathrm{kPa}$. The numerical results are shown in Figures 16-18, with the original position of the beam indicated with an outline. The final resting position of the first simulation is shown in Figure 16. The response clearly falls in regime 1, attaining large permanent deformation without fracturing. The position of the beam in the second simulation $(17.8 \mathrm{kPa} \mathrm{s})$ shortly after the moment of fracture is shown in Figure 17. The response belongs to the second regime: the beam fails at the supports after the out-of-plane deflection at the center of the span has reached approximately $11 \mathrm{~mm}$ (nearly twice the beam thickness). The beam in the third simulation (39.6 kPa) fails immediately, without any significant plastic deformation (Figure 18). This failure is clearly shear-dominated. We note that the agreement of the simulations is only qualitative at this point; a characterization of the plasticity and fracture properties of the material is necessary to obtain a quantitative agreement. However, the ability of the proposed simulation framework to detect the competition between the in-plane tensile failure mode and the transverse shear mode is evident.

\subsection{Scalability test}

The parallel scalability of the computational framework and our implementation is examined in this section. The tests were conducted on a cluster containing nodes each with two Intel $2.26 \mathrm{GHz}$ Xeon E5520 quad-core processors and 24 GB of memory. The nodes are connected with a 4x DDR Infiniband network. The test problem is the free oscillation of a cantilever beam.

The first test is a weak scalability test, in which the problem size and the number of processors are increased proportionally so as to maintain a constant computational load per processor. The base problem is defined with a structured right triangular mesh, having 200 elements in the long direction of the beam, and 40 elements across the width, for a total of 16000 elements. This corresponds to $384 \times 10^{3}$ degrees of freedom, ignoring degrees of freedom removed by essential boundary conditions. The load per processor is 
Figure 16: Beam blast simulation, Case 1: $10.9 \mathrm{kPa}$ s impulse. Final static position of the beam. The outline represents the initial configuration.

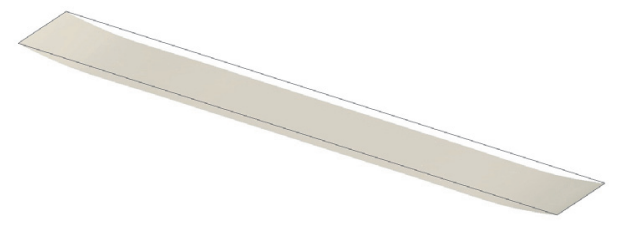

(a)

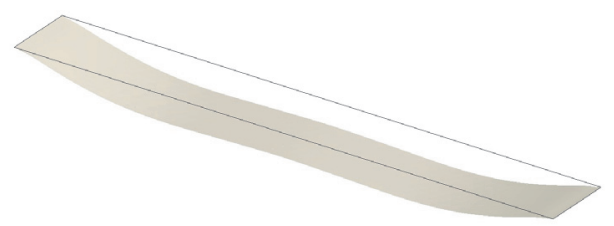

(c)

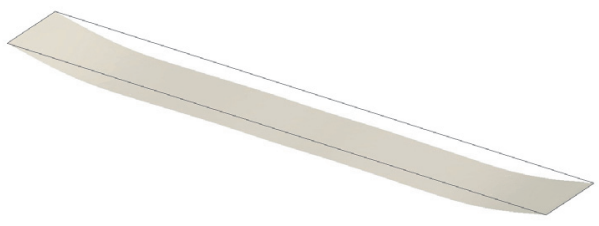

(b)

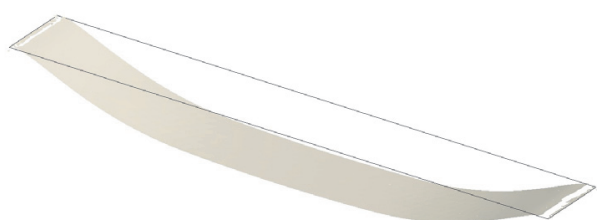

(d)

Figure 17: Beam blast simulations, Case 2: $17.8 \mathrm{kPa}$ s impulse. The outline indicates the initial position of the beam. (a)-(d) show successive snapshots of the response though time. 


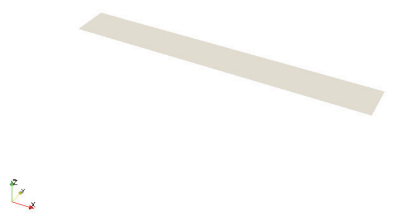

(a)

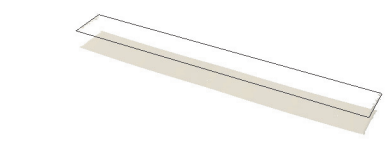

(b)

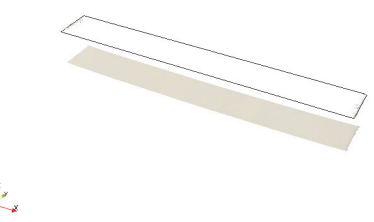

(c)

Figure 18: Beam blast simulations, Case 3: $39.6 \mathrm{kPa} \mathrm{s}$ impulse. (a)-(c) show successive snapshots of the response through time. The outline indicates the initial position of the beam. The beam shears off at the supports almost immediately without any perceivable bending deformation.

kept fixed between succesive simulations by uniformly subdividing the mesh and increasing the number of processors by a factor of 4 . For each simulations, the problem was integrated forward in time through 100 time steps and the mean computation time per simulation time step was measured. The results are shown in Figure 19. The plot shows the computation time per simulation time step plotted against the number of processors. Perfect weak scalability would appear as a horizontal line. The scalability of the present method implementation is seen to be quite good.

We also applied a strong scalability test, wherin the total problem size is held constant and the number of processors is increased. CPU time per integration step was measured on three problems of differing sizes: the first was the same as the base mesh in the weak scalability test, and the other two were obtained by successive uniform subdivisions of this mesh. Thus, the largest problem contained $256 \times 10^{3}$ elements and $6.1 \times 10^{6}$ degrees of freedom. The problem was again integrated forward through 100 time steps, with the mean computation time per integration step shown in Figure 19. Ideal scalability is indicated by the grey triangle in the figure. The implementation shows good strong scalability until the number of elements per processor drops below 1000. At this point the communication overhead becomes significant compared to the computation time.

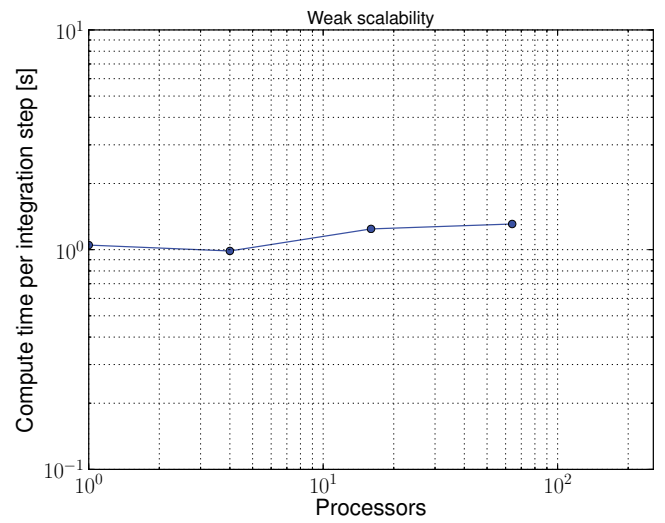

(a)

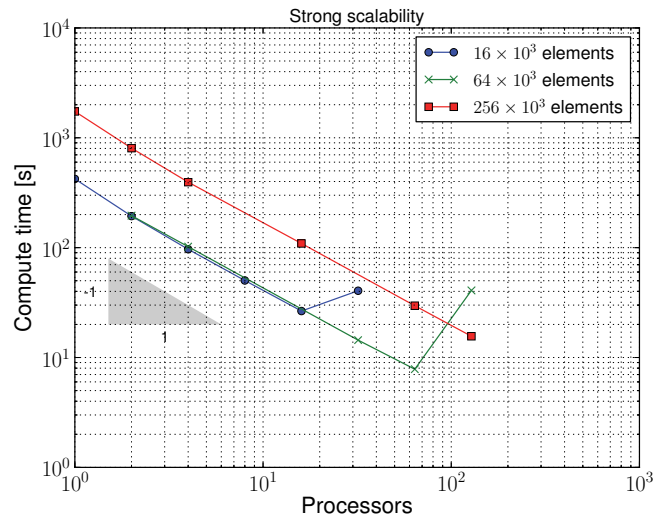

(b)

Figure 19: (a) Measurements from weak scalability test. The problem size is 16,000 elements per processor. (b) Measurements from the strong scalability test.

\subsection{Explosive decompression in a commercial airliner}

This example demonstrates the capabilities of the simulation framework to handle widespread failure and fragmentation in a large structure. We simulate an idealized explosive decompression in a commercial 
airliner. The computational mesh corresponds to the outer skin of an Airbus A319 and is provided by an example in the distribution of Gmsh [39]. For simplicity, we do not model the interior beam framework or any other structural elements of the fuselage. The original mesh has been uniformly subdivided, giving a mesh that comprises approximately 650000 quadratic triangle elements, with an average element edge length of $5 \mathrm{~cm}$. The mesh is shown in Figure 20. The computational domain is partitioned among 208 processors as illustrated in Figure 20. A portion of the skin is subjected to a time-dependent, spatially uniform impulsive pressure on the region identified in Figure 21 according to the equation ${ }^{6}$

$$
p(t)=p_{0} \exp \left(-t / t_{p}\right)\left(1-\frac{t}{t_{p}}\right)
$$

The parameters of the problem are given in Table 4.

Table 4: Parameters used for the simulation of an explosive decompression of an airliner fuselage.

\begin{tabular}{ccccccccc}
\hline$E$ & $\nu$ & $h$ & $\rho^{0}$ & $Y_{0}$ & $H$ & $\sigma_{c}$ & $\Gamma$ \\
\hline $72 \mathrm{MPa}$ & 0.34 & $1.5 \mathrm{~mm}$ & $2700 \mathrm{~kg} / \mathrm{m}^{3}$ & $490 \mathrm{MPa}$ & $933 \mathrm{MPa}$ & $540 \mathrm{MPa}$ & $3.2 \mathrm{MJ} / \mathrm{m}^{2}$ \\
\hline \multicolumn{10}{c}{$\alpha_{1}$} & $\alpha_{2}$ & $\alpha_{3}$ & $c_{x}$ & $c_{T}$ & $p_{0}$ & $t_{p}$ \\
\hline & & 1 & 1 & 3 & 3 & $3.37 \mathrm{MPa}$ & $692 \mu \mathrm{s}$ \\
\hline
\end{tabular}

Simulated deformations of the fuselage are shown in Figures 22 and 23. The simulation framework captures a complex array of effects, including wrinkling and dynamic buckling, "petalling" type fracture, and the formation of free fragments that continue to move inertially once free from the structure.

\section{Summary and Conclusions}

We have presented a framework for the simulation of dynamic deformation and fracture in shell structures. The framework is composed of a cohesive fracture model embedded within a discontinuous Galerkin shell discretization. We have shown that it is possible to take advantage of the characteristics of the discontinuous Galerkin method to simulate fracture in a scalable way, while avoiding the introduction of artificial elastic compliance in the cohesive law. The scalability of the framework makes it a powerful tool to address massive damage and impulsive loading problems in large structures. As evidence of this, we demonstrated an example of explosive decompression in the outer skin of a full-scale passenger aircraft.

The proposed framework is based on nonlinear shear-flexible shell theory, which permits the simulation of the through-thickness shearing fracture mode that is not possible with thin shell (Kirchhoff-Love) shell kinematics. We have demonstrated that the framework is capable of representing this through-thickness shearing mode with numerical examples. In particular, we simulated the beam blast experiments of Menkes and Opat, which illustrate a change in fracture mode from in-plane tearing to shear-off as the impulse increases, and showed that our framework is capable of detecting this effect.

Finally, we note some limitations of the framework. The shear-flexible shell formulation introduces an additional kinematic field (the director vector) with respect to Kirchhoff-Love shells, and is thus more expensive. Therefore, some efficiency can be achieved with Kirchhoff-Love shells if the shear fracture mode is not expected. The discontinuous Galerkin discretization also increases the number of degrees of freedom relative to standard continuous finite element interpolations of the same polynomial degree; however, this drawback is mitigated by the scalability of the method.

\footnotetext{
6 This pressure history is not representative of any particular real-world scenario.
} 


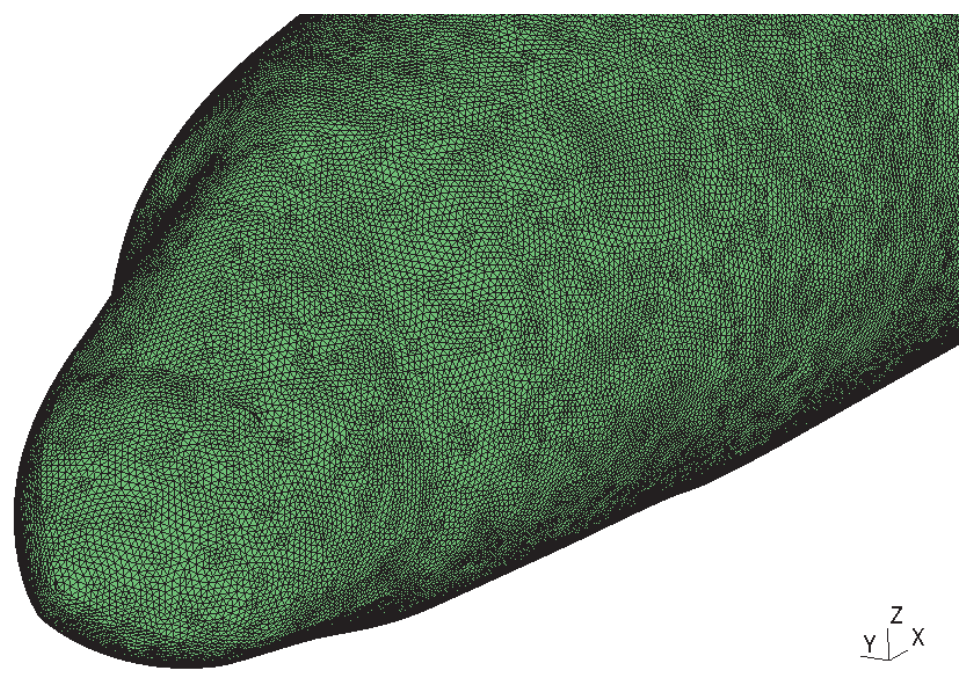

(a)

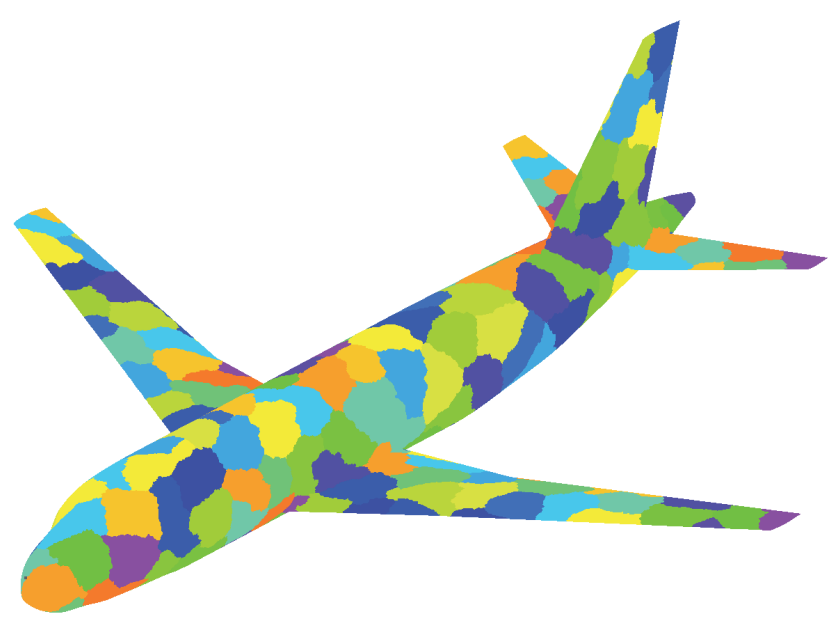

$x^{2} x$

(b)

Figure 20: Computational mesh used of the Airbus A319 fuselage. (a) Close-up of the nose. All elements in the mesh are roughly $5 \mathrm{~cm}$ in edge length. The mesh contains approximately 650000 elements and $15 \times 10^{6}$ total degrees of freedom. (b) Map of the domain decomposition for parallel simulation. Each colored region is a processor sub-domain. The mesh is partitioned into 208 sub-domains, each containing approximately 3000 elements. 


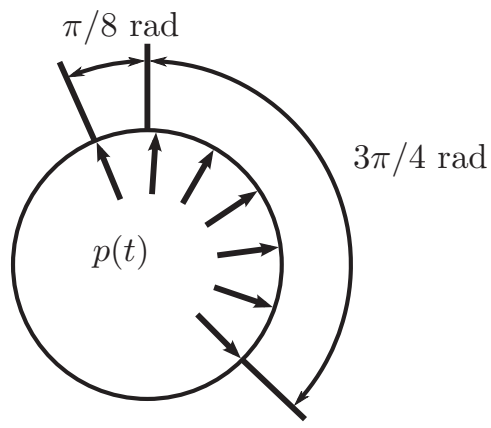

(a)

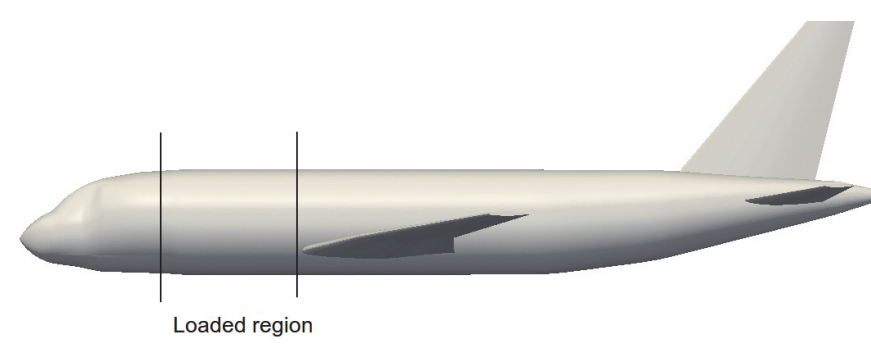

(b)

Figure 21: Spatial location of pressure load for simulation of explosive decompression of an airliner fuselage. (a) Schematic of the fuselage cross-section showing the angular azimuthal extents of the loaded region. The angles are measured from the vertical along the diameter. (b) Side view of the fuselage showing the axial extents of the loaded region.

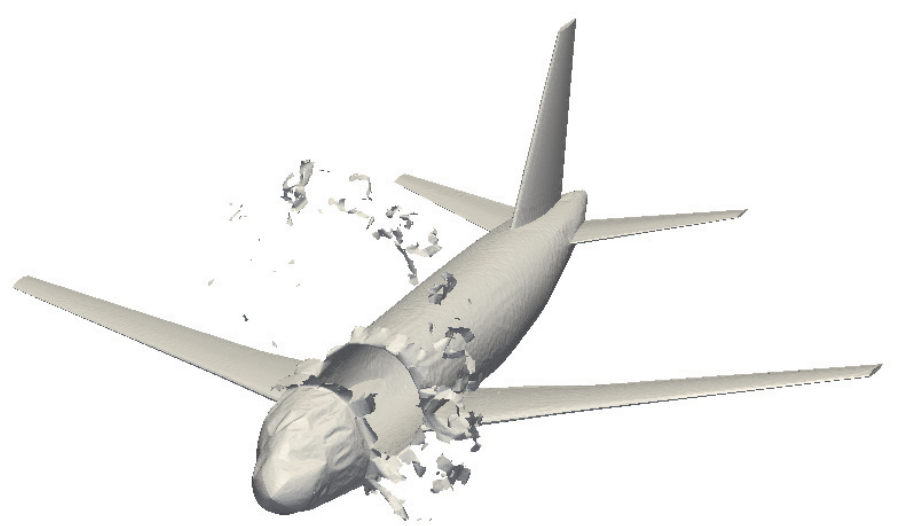

Figure 22: State of the fuselage at $t=9.907 \mathrm{~ms}$. Free-flying fragments and petalling of the skin near the edges of the loaded region are visible. 


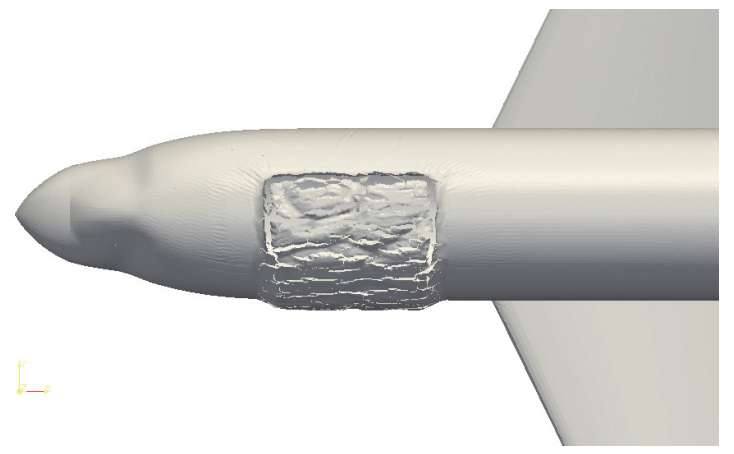

(a) $t=0.977 \mathrm{~ms}$

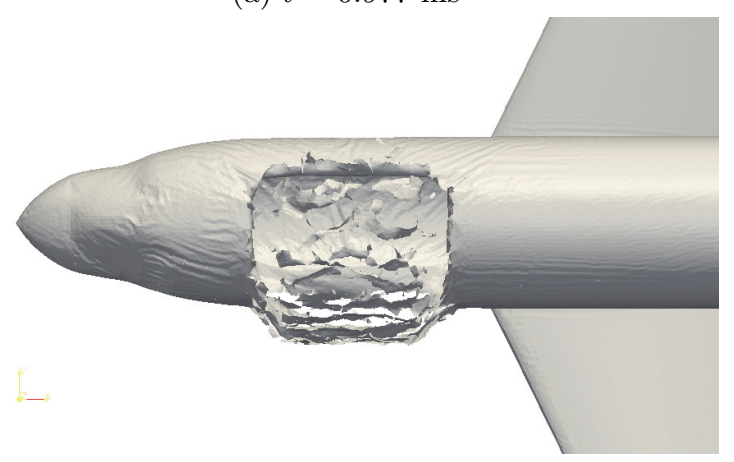

(c) $t=2.899 \mathrm{~ms}$

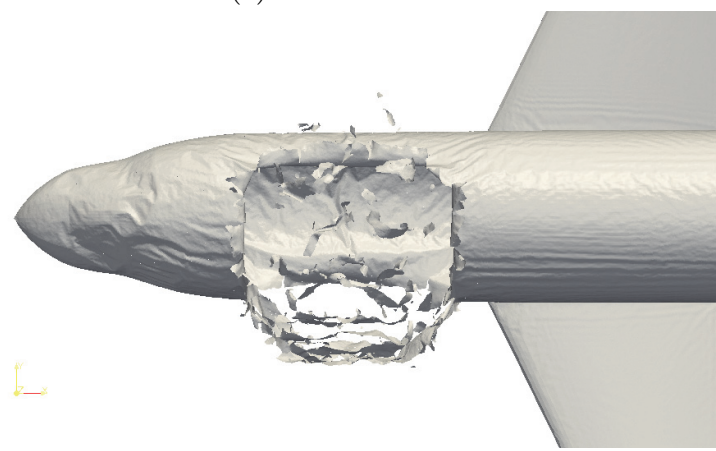

(e) $t=4.852 \mathrm{~ms}$

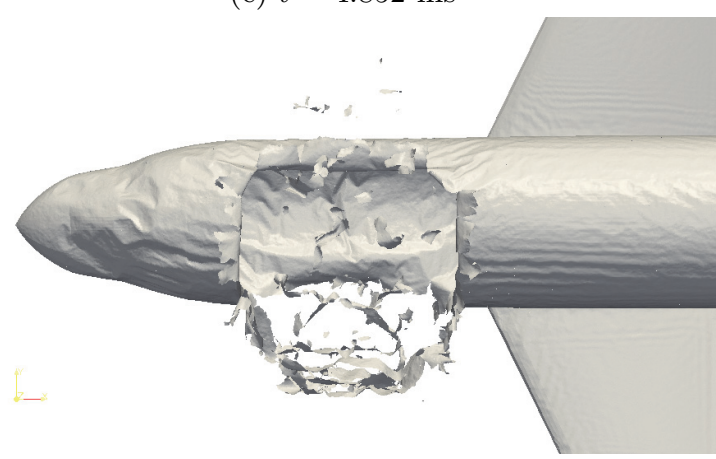

(g) $t=6.806 \mathrm{~ms}$

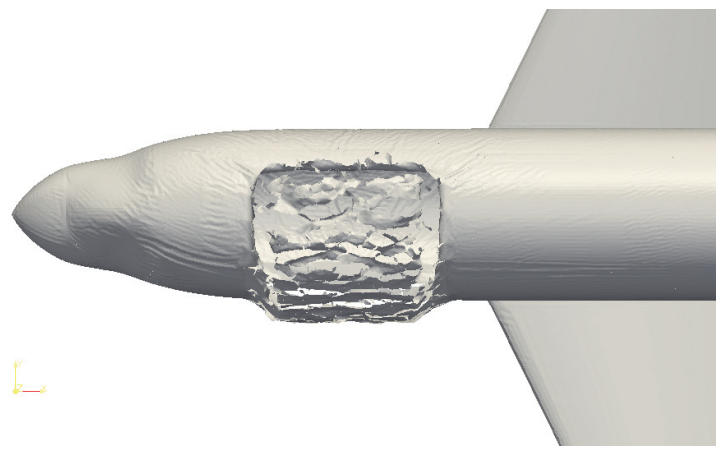

(b) $t=1.922 \mathrm{~ms}$

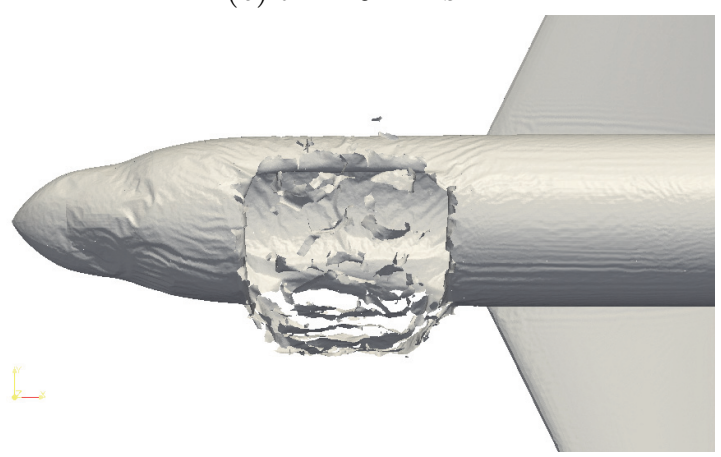

(d) $t=3.876 \mathrm{~ms}$

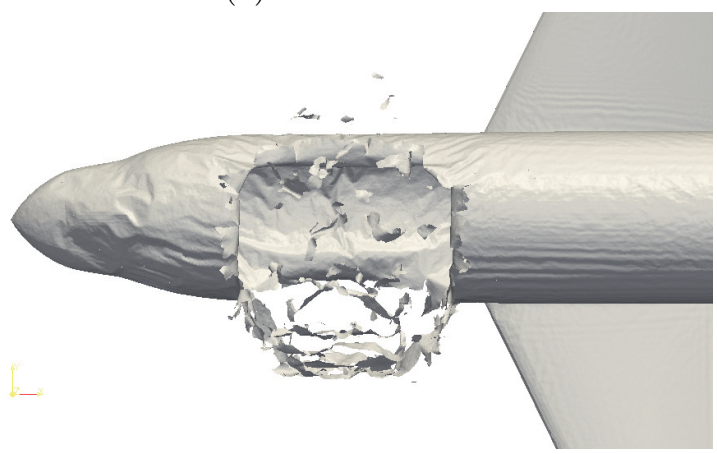

(f) $t=5.829 \mathrm{~ms}$

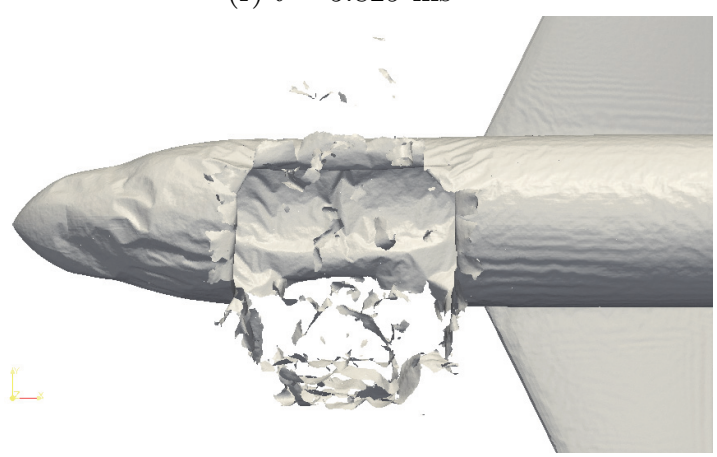

(h) $t=7.783 \mathrm{~ms}$

Figure 23: Snapshots of simulation results for the explosive decompression of an airliner fuselage. Dynamic buckling waves travelling from the loaded region are visible, as well as complex skin wrinkling patterns. 


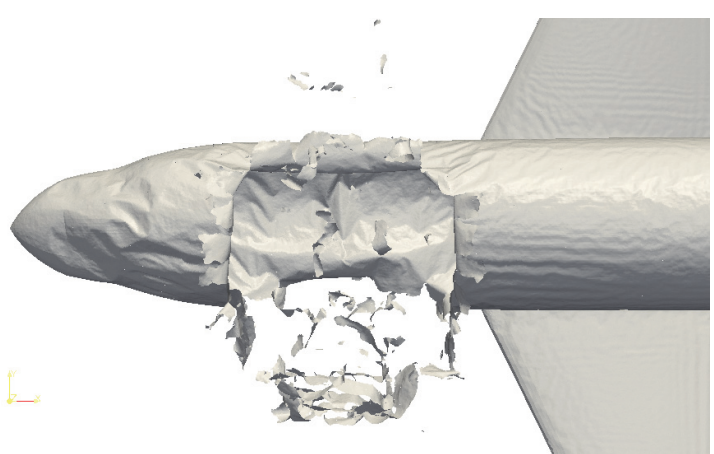

(i) $t=8.737 \mathrm{~ms}$

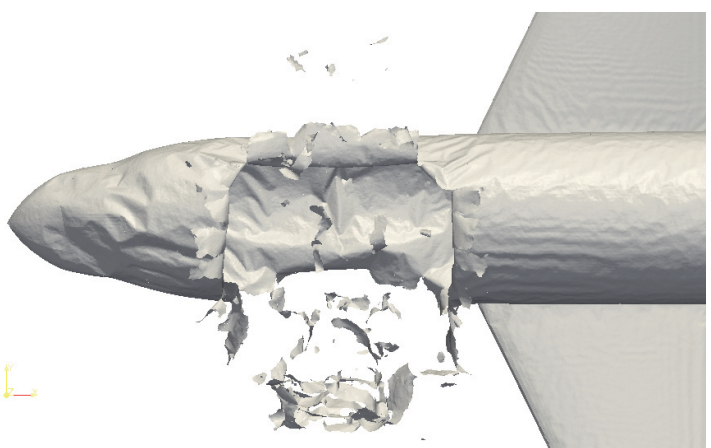

(j) $t=9.712 \mathrm{~ms}$

Figure 23: Snapshots of simulation results for the explosive decompression of an airliner fuselage.

\section{Acknowledgements}

This research was supported by the U.S. Army through the Institute for Soldier Nanotechnologies, under contract W911NF-13-D-0001 with the U.S. Army Research Office, and by the Office of Naval Research (ONR grant number N00014-07-1-0764) as part of a Multidisciplinary University Research Initiative (David Shifler, Program Manager). The content does not necessarily reflect the position of the government, and no official endorsement should be inferred.

\section{Appendix A. Summary of the representation of the director field}

The initial rotation tensor at each director node is computed by recourse to Rodrigues' formula:

$$
\left.\boldsymbol{\Lambda}_{A}^{0}=\left(\mathbf{t}_{A}^{0} \cdot \mathbf{E}_{3}\right) \mathbf{1}+\widehat{\left(\mathbf{E}_{3} \times \mathbf{t}_{A}^{0}\right.}\right)+\frac{1}{1+\mathbf{t}_{A}^{0} \cdot \mathbf{E}_{3}}\left(\mathbf{E}_{3} \times \mathbf{t}_{A}^{0}\right) \otimes\left(\mathbf{E}_{3} \times \mathbf{t}_{A}^{0}\right)
$$

The operation $\widehat{\bullet}: \mathbb{R}^{3} \rightarrow s o(3)$ gives the cross product tensor associated to a vector. ${ }^{7}$ The nodal rotation tensors are updated in an incremental fashion as the shell deforms through the use of the exponential map on the unit sphere. Remarkably, this can be written in closed form. This update procedure was given by Simo et al. [23], and is summarized in Algorithm 1 for completeness. This algorithm is geometrically exact, and well-conditioned for increments of arbitrarily large size.

[1] G. I. Barenblatt, The formation of equilibrium cracks during brittle fracture. general ideas and hypotheses. axiallysymmetric cracks., Journal of Applied Mathematics and Mechanics 23 (3) (1959) 622-636.

[2] G. Barenblatt, The mathematical theory of equilibrium cracks in brittle fracture, Advances in Applied Mechanics 7 (1962) $55-129$.

[3] M. Ortiz, S. Suresh, Statistical properties residual stresses and intergranular fracture in ceramic materials, Journal of Applied Mechanics 60 (1993) 77-84.

[4] G. Camacho, M. Ortiz, Computational modeling of impact damage in brittle materials, International Journal of Solids and Structures 33 (20-22) (1996) 2899-2983.

[5] A. Seagraves, R. Radovitzky, Dynamic Failure of Materials and Structures, Springer, 2009, Ch. 12 Advances in Cohesive Zone Modeling of Dynamic Fracture, pp. 349-405.

[6] F. Cirak, M. Ortiz, A. Pandolfi, A cohesive approach to thin-shell fracture and fragmentation, Computer Methods in Applied Mechanics and Engineering 194 (2005) 2604-2618.

[7] P. Zavattieri, Modeling of crack propagation in thin-walled structures using a cohesive model for shell elements, Journal of Applied Mechanics 73 (2006) 948-958.

${ }^{7}$ That is, for an arbitrary $\mathbf{a} \in \mathbb{R}^{3}, \widehat{\mathbf{a}}$ is the tensor with the matrix representation in the canonical basis

$$
[\widehat{\mathbf{a}}]=\left[\begin{array}{ccc}
0 & -a^{3} & a^{2} \\
a^{3} & 0 & -a^{1} \\
-a^{2} & a^{1} & 0
\end{array}\right]
$$




Algorithm 1 Kinematic update procedure.

1: Compute spatial director increments

$$
\Delta \mathbf{t}_{A}=\bar{\Lambda}_{A}^{n} \Delta \mathbf{T}_{A}
$$

2: Update directors with exponential map

$$
\mathbf{t}_{A}^{n+1}=\exp _{\mathbf{t}_{A}^{n}}\left(\Delta \mathbf{t}_{A}\right):=\cos \left\|\Delta \mathbf{t}_{A}\right\| \mathbf{t}_{A}^{n}+\frac{\sin \left\|\Delta \mathbf{t}_{A}\right\|}{\left\|\Delta \mathbf{t}_{A}\right\|} \Delta \mathbf{t}_{A}
$$

3: Compute rotation increment

$$
\Delta \boldsymbol{\Lambda}_{A}=\cos \left\|\Delta \mathbf{t}_{A}\right\| \mathbf{1}+\frac{\sin \left\|\Delta \mathbf{t}_{A}\right\|}{\left\|\Delta \mathbf{t}_{A}\right\|}\left(\mathbf{t}_{A}^{n \times \Delta \mathbf{t}_{A}}\right)+\frac{1-\cos \left\|\Delta \mathbf{t}_{A}\right\|}{\left\|\Delta \mathbf{t}_{A}\right\|^{2}}\left(\mathbf{t}_{A}^{n} \times \Delta \mathbf{t}_{A}\right) \otimes\left(\mathbf{t}_{A}^{n} \times \Delta \mathbf{t}_{A}\right)
$$

4: Update rotations

$$
\boldsymbol{\Lambda}_{A}^{n+1}=\Delta \boldsymbol{\Lambda}_{A} \boldsymbol{\Lambda}_{A}^{n}
$$

[8] B. L. Talamini, R. A. Radovtizky, A discontinuous Galerkin method for nonlinear shear-flexible shells, Computer Methods in Applied Mechanics and Engineering 303 (2016) 128-162.

[9] J. Mergheim, E. Kuhl, P. Steinmann, A hybrid discontinuous Galerkin/interface method for the computational modelling of failure, Communication in Numerical Methods in Engineering 20 (2004) 511-519.

[10] R. Radovitzky, A. Seagraves, M. Tupek, L. Noels, A scalable 3D fracture and fragmentation algorithm based on a hybrid, discontinuous Galerkin, Cohesive Element Method, Computer Methods in Applied Mechanics and Engineering 200 (2011) 326-344.

[11] I. Babuška, M. Zlámal, Nonconforming elements in the finite element method with penalty, SIAM Journal of Numerical Analysis 10 (5) (1973) 863-875.

[12] H. Espinosa, P. Zavattieri, A grain level model for the study of failure initiation and evolution in polycrystalline brittle materials. Part I: Theory and numerical implementation, Mechanics of Materials 35 (2003) 333-364.

[13] G. Becker, C. Geuzaine, L. Noels, A one field full discontinuous Galerkin method for Kirchhoff-Love shells applied to fracture mechanics, Computer Methods in Applied Mechanics and Engineering 200 (45-46) (2011) 3223-3241.

[14] G. Becker, L. Noels, A full-discontinuous Galerkin formulation of nonlinear Kirchhoff-Love shells: elasto-plastic finite deformations, parallel computation, and fracture applications, International Journal for Numerical Methods in Engineering (2013) 80-117.

[15] S. B. Menkes, H. J. Opat, Broken beams, Experimental mechanics 13 (11) (1973) 480-486.

[16] I. Scheider, W. Brocks, Cohesive elements for thin-walled structures, Computational Materials Science 37 (2006) $101-109$.

[17] I. Scheider, M. Rajendran, A. Banerjee, Comparison of different stress-state dependent cohesive zone models applied to thin-walled structures, Engineering Fracture Mechanics 78 (2011) 534-543.

[18] K. Nielsen, J. Hutchinson, Cohesive traction-separation laws for tearing of ductile metal plates, International Journal of Impact Engineering 48 (2012) 15-23.

[19] P. B. Woelke, M. D. Shields, N. N. Abboud, J. W. Hutchinson, Simulations of ductile fracture in an idealized ship grounding scenario using phenomenological damage and cohesive zone models, Compuational Materials Science 80 (2013) $79-95$.

[20] P. Woelke, M. Shields, J. Hutchinson, Cohesive zone modeling and calibration for mode I tearing of large ductile plates, Engineering Fracture Mechanics 147 (2015) 293-305.

[21] J. Simo, D. Fox, On a stress resultant geometrically exact shell model. Part I: formulation and optimal parametrization, Computer Methods in Applied Mechanics and Engineering 72 (1989) 267-304.

[22] K. Papoulia, C.-H. Sam, S. Vavasis, Time continuity in cohesive finite element modeling, International Journal for Numerical Methods in Engineering 58 (2003) 679-701.

[23] J. Simo, D. Fox, M. Rifai, On a stress resultant geometrically exact shell model. Part III: computational aspects of the non-linear theory, Computer Methods in Applied Mechanics and Engineering 79 (1990) 21-70.

[24] D. Arnold, F. Brezzi, B. Cockburn, L. Marini, Unified analysis of discontinuous Galerkin methods for elliptic problems, SIAM Journal on Numerical Analysis 39 (5) (2002) 1749-1779.

[25] A. Ten Eyck, A. Lew, Discontinuous Galerkin methods for nonlinear elasticity, International Journal for Numerical Methods in Engineering 67 (2006) 1204-1243.

[26] J. Simo, M. Rifai, D. Fox, On a stress resultant geometrically exact shell model. Part VI: Conserving algorithms for non-linear dynamics, International Journal for Numerical Methods in Engineering 34 (1992) 117-164.

[27] B. Brank, S. Mamouri, A. Ibrahimbegović, Constrained finite rotations in dynamics of shells and Newmark implicit 
time-stepping schemes, Engineering Computations 22 (2005) 505-535.

[28] C. Sansour, P. Wriggers, J. Sansour, Nonlinear dynamics of shells: Theory, finite element formulation, and integration schemes, Nonlinear Dynamics 13 (3) (1997) 279-305.

[29] T. J. R. Hughes, The finite element method: Linear static and dynamic finite element analysis, Dover Publications, Inc, New York, 2000

[30] L. Noels, R. Radovitzky, An explicit discontinuous Galerkin method for non-linear solid dynamics. Formulation, parallel implementation and scalability properties., International Journal for Numerical Methods in Engineering 74 (9) (2007) 1393-1420.

[31] E. Hinton, T. Rock, O. Zienkiewicz, A note on mass lumping and related processes in the finite element method, Earthquake Engineering and Structural Dynamics 4 (1976) 245-249.

[32] G. Karypis, V. Kumar, A fast and highly quality multilevel scheme for partitioning irregular graphs, SIAM Journal of Numerical Analysis 20 (1) (1999) 359-392.

[33] J. Simo, J. Kennedy, On a stress resultant geometrically exact shell model. Part V: Nonlinear plasticity: formulation and integration algorithms, Computer Methods in Applied Mechanics and Engineering 96 (1992) 133-171.

[34] J. Dujc, B. Brank, Stress resultant plasticity for shells revisited, Computer Methods in Applied Mechanics and Engineering 247 (2012) 146-165.

[35] L. Morino, J. W. Leech, E. A. Witmer, An improved numerical calculation technique for large elastic-plastic transient defomations of thin shells: Part 2-evaluation and applications, Journal of Applied Mechanics 38 (2) (1971) 429-436.

[36] T. Belytschko, J. Lin, C. Tsay, Explicit algorithms for the nonlinear dynamics of shells, Computer Methods in Applied Mechanics and Engineering 42 (1984) 225-251.

[37] J.-H. Yan, M. A. Sutton, X. Deng, Z. Wei, P. Zavattieri, Mixed-mode crack growth in ductile thin-sheet materials under combined in-plane and out-of-plane loading, International Journal of Fracture 160 (2009) 169-188.

[38] Y. Li, T. Wierzbicki, M. Sutton, J. Yan, X. Deng, Mixed mode stable tearing of thin sheet Al 6061-T6 specimens: experimental measurements and finite element simulations using a modified Mohr-Coulomb fracture criterion, International Journal of Fracture 168 (2011) 53-71.

[39] C. Geuzaine, J. Remacle, Gmsh: A 3-d finite element mesh generator with built-in pre- and post-processing facilities, International Journal for Numerical Methods in Engineering 79 (2009) 1309-1331. 